\title{
MAPPINGS OF A NORMAL SPACE INTO AN ABSOLUTE NEIGHBORHOOD RETRACT
}

\author{
BY \\ SZE-TSEN HU
}

\section{InTRODUCTION}

Given two topological spaces $X$ and $Y$, let us consider the totality of the mappings (that is, continous transformations) $f: X \rightarrow Y$. These mappings are divided into homotopy classes, those in each class being homotopic to each other. The problem of determining these classes by means of known invariants of $X$ and $Y$ is of great importance in modern topology.

For the most interesting special case where $Y=S^{n}$, an $n$-sphere, Hopf's brilliant results and their various generalizations solve the problem completely, if $X$ is a compact (= bicompact) Hausdorff space with(1) $\operatorname{dim} X \leqq n$. As newly formulated by Alexandroff $[1$, p. 17$]\left({ }^{2}\right)$, this theorem may be stated as follows:

HOPF CLASSIFICATION THEOREM. The homotopy classes of the mappings of a compact Hausdorff space $X$ with $\operatorname{dim} X \leqq n$ into an $n$-sphere $S^{n}$ are in a (1-1)correspondence with the elements of the n-dimensional Cech cohomology group $H^{n}(X)$ with integer coefficients. The correspondence is determined by the operation $f \rightarrow f^{*}\left(s^{n}\right)$, where $s^{n}$ is a generator of the cohomology group $H^{n}\left(S^{n}\right)$ with integer coefficients and $f^{*}: H^{n}\left(S^{n}\right) \rightarrow H^{n}(X)$ is the homomorphism induced by the mapping $f: X \rightarrow S^{n}$.

On the other hand, in the case where $X$ is a geometric complex, finite or infinite, and $Y$ a topological space, Eilenberg [9] gave some far reaching general theorems on the extension and homotopy of these mappings, which include the original Hopf theorems as mere particular cases. These beautiful theorems of Eilenberg can not be generalized to the case where $X$ is a topological space, unless some suitable restrictions are to be put either on $X$ or on $Y$. A glance at the Hopf classification theorem given above will justify our preference of restriction to $Y$ instead of $X$.

The object of the present work is to build up a generalization of Eilenberg's theory under the assumptions that $X$ be a normal space $[14$, p. 26] and that $Y$ be an absolute neighborhood retract (ANR) $[15$, p. 58], which includes the generalized Hopf theorems as special cases. Owing to the Cech

Presented to the Society, December 31, 1947; received by the editors August 2, 1947.

(1) For a given topological space $X$, by $\operatorname{dim} X \leqq n$ we mean that any finite open covering of $X$ has a refinement whose nerve is of dimension not exceeding $n$.

(2) Numbers in brackets refer to the bibliography at the end of the paper. 
cohomology theory, the undesirable condition of compactness has to be assumed in the most of the paper; however, it can clearly be removed by using a cohomology theory based on more general coverings as Dowker [6] did.

The general trend of the recent years is that most of the topologists prefer cohomology to homology. This is probably because cohomology of ten simplifies both the statement and the proof of many a topological theorem. Since cohomology is no longer less intuitive than homology nowadays, homological interpretations of theorems termed naturally in cohomology seem to be mere redundancies. On this account, no homology will be mentioned in the present paper.

Preliminaries of our treatment are given in Part II. $\$ 1$ gives a very brief sketch of the Cech cohomology sequence, a knowledge of which will be needed in the sequel. $\$ 2$ presents the fundamental bridge theorems which are the main tools of this investigation.

The general theory is developed in Part III as follows: Let $X_{0}$ be a closed set of $X$. The notion of $n$-extensibility of the mappings $f: X_{0} \rightarrow Y$ is defined in $\S 3$; and the obstruction sets $\Omega^{n+1}(f)$ of an arbitrary mapping $f: X_{0} \rightarrow Y$ are introduced in $\$ 4$. All of them are invariants under a homotopy; and by the aid of them our general extension theorems are established in $\$ 5$. On the other hand, in $\$ 6$ we introduce the notion of $n$-homotopy between two given mappings $f_{0}, f_{1}: X \rightarrow Y$; and in $\S 7$ we define their separation sets $\Delta^{n}\left(f_{0}, f_{1}\right)$. They depend only on the homotopy classes of the given mappings; and by the aid of them our general homotopy theorems are formulated in §8. Settheoretic meanings of both the $n$-extensibility and the $n$-homotopy are given in their respective sections.

Part IV contains the complete solution for the case where $X$ is a compact Hausdorff space and $Y$ is an ANR satisfying $\pi^{r}(Y)=0$ for each $r<n$ together with some minor conditions. In this case, the characteristic element $\kappa^{n}(f)$ of a mapping $f$ can be defined ( $\$ 9)$; and thus a complete generalization of the Hopf classification theorem is obtained in $\$ 11$. Further, if $Y$ is compact we introduce the basic element $\iota^{n}(Y)$ of $Y$ in $\$ 12$ as an analogue of the element determined by the fundamental $n$-cocycle of an $n$-sphere $S^{n}$, which enables us to formulate our generalized Hopf classification theorem exactly parallel to the one given above for $Y=S^{n}$. An application of this formulation is given in $\$ 13$ to establish for compact ANR the theorems concerning homotopy types, proved by Eilenberg [10, p. 464] only for geometric complexes.

\section{Preliminaries}

1. Čech cohomology sequence. In their axiomatic approach to the homology theory, Eilenberg and Steenrod [11] formulated the homology sequence (called by them the natural system of the homology theory) as an axiom which should be satisfied in all admissible homology theories. The sequence given in this comprehensive form is a weapon far more powerful than the 
classical duality theorem of Alexander. Since no detailed exposition of the Cech cohomology sequence has appeared in print, a brief sketch seems to be desirable for the convenience and clearness of the applications in this paper.

Let $X$ denote an arbitrary topological space, $X_{0}$ a closed subset of $X$, and $X_{*}=X-X_{0}$ its open complement. Following Lefschetz, we shall call $\left(X_{0}, X_{*}\right)$ a dissection of $X$. Let $G$ denote a discrete abelian group written additively.

A finite open covering will be simply called a covering. Let $\subseteq$ denote the set of all coverings of $X$, partially ordered by the statement $\alpha<\beta$ if $\beta$ is a refinement of $\alpha[14$, p. 13]. $\subseteq$ is a reflexive directed set $[14$, p. 4].

Let $\alpha=\left\{a_{1}, a_{2}, \cdots, a_{r}\right\}$ be a covering of $X$ and let $A$ denote the nerve of $\alpha\left[14\right.$, p. 244]. The nonvoid sets of $X_{0} \cap a_{i}(i=1,2, \cdots, r)$ form a covering $\alpha_{0}$ of $X_{0}$ whose nerve $A_{0}$ is a closed subcomplex of $A$. Let $A_{*}$ denote the open complement $A-A_{0}$. Let us denote by $H^{n}(A, G), H^{n}\left(A_{0}, G\right), H^{n}\left(A_{*}, G\right)$ the $n$-dimensional cohomology groups of the complexes $A, A_{0}, A_{*}$ respectively. We shall use these notations as typical ones for the coverings $\alpha, \beta, \cdots$ of $X$.

Suppose $\alpha<\beta$, that is, let $\beta$ be a refinement of $\alpha$. Let us select for each member of $\beta$ a member of $\alpha$ containing it. This gives a simplicial mapping $p_{\beta \alpha}: B \rightarrow A$, called a simplicial projection. Clearly $p_{\beta \alpha}\left(B_{0}\right) \subset A_{0}$. All the possible simplicial projections $p_{\beta \alpha}$ induce uniquely the homomorphisms of the cohomology groups of $A, A_{0}, A_{*}$ into those of $B, B_{0}, B_{*}$ respectively, which will be denoted by $h_{\alpha \beta}$. If $\alpha<\beta<\gamma$, then clearly $p_{\beta \alpha} p_{\gamma \beta}$ is a simplicial projection $p_{\gamma \alpha}$. Hence

$$
\left\{H^{n}(A, G)\right\}, \quad\left\{H^{n}\left(A_{0}, G\right)\right\}, \quad\left\{H^{n}\left(A_{*}, G\right)\right\}
$$

are direct systems of groups with $h_{\alpha \beta}$ as the homomorphisms [14, p. 57].

By the $n$-dimensional Čech cohomology group $H^{n}(X, G)$ of $X$ with coefficients in $G$ we mean the limit group of the direct system $\left\{H^{n}(A, G)\right\}$. The limit group of the direct system $\left\{H^{n}\left(A_{*}, G\right)\right\}$ is defined to be the $n$-dimensional Cech cohomology group of $X$ modulo $X_{0}$ with coefficients in $G$, denoted by $H^{n}\left(X \bmod X_{0}, G\right)$. The isomorphism

$$
H^{n}\left(X_{0}, G\right) \approx \lim \left\{H^{n}\left(A_{0}, G\right)\right\}
$$

is obvious; but the groups $H^{n}\left(X \bmod X_{0}, G\right)$ and $H^{n}\left(X_{*}, G\right)$ need not be isomorphic. This justifies the notations we used.

For each $n \geqq 0$ there are unique homomorphisms

$$
\begin{array}{ll}
\pi: & H^{n}\left(X \bmod X_{0}, G\right) \rightarrow H^{n}(X, G), \\
\eta: & H^{n}(X, G) \rightarrow H^{n}\left(X_{0}, G\right), \\
\delta: & H^{n}\left(X_{0}, G\right) \rightarrow H^{n+1}\left(X \bmod X_{0}, G\right)
\end{array}
$$

described as follows. For each covering $\alpha$ of $X$, the projection $A \rightarrow A_{*}$ and the injection $A_{0} \rightarrow A$ induce a homomorphism $\pi_{\alpha}$ of the cocycles of $A_{*}$ into those of $A$ and a homomorphism $\eta_{\alpha}$ of the cocycles of $A$ into those of $A_{0}$ 
$\left[14\right.$, p. 113]. Following Whitney, we shall denote by $\delta_{\alpha}$ the coboundary operator in $A$. Let $\xi \in H^{n}\left(X \bmod X_{0}, G\right)$ be an arbitrary element represented by a cocycle $z^{n}$ in $A_{*}$ for some covering $\alpha$ of $X$; then $\pi(\xi) \in H^{n}(X, G)$ is the element represented by the cocycle $\pi_{\alpha}\left(z^{n}\right)$, which is actually $z^{n}$ considered as a cocycle of $A$. If $\xi \in H^{n}(X, G)$ be an arbitrary element represented by a cocycle $z^{n}$ in $A$ for some covering $\alpha$ of $X$; then $\eta(\xi) \in H^{n}\left(X_{0}, G\right)$ is the element represented by the cocycle $\eta_{\alpha}\left(z^{n}\right)$ of $A_{0}$, which is actually the part of $z^{n}$ on $A_{0}$. Let $\xi \in H^{n}\left(X_{0}, G\right)$ be an arbitrary element represented by a cocycle $z^{n}$ of $A_{0}$ for some covering $\alpha$ of $X$; then $\delta(\xi) \in H^{n+1}\left(X \bmod X_{0}, G\right)$ is the element represented by the cocycle $\delta_{\alpha}\left(z^{n}\right)$ in $A_{*}$.

The theorem of Cech cohomology sequence of the dissection $\left(X_{0}, X_{*}\right)$ is stated as follows:

(1.1) Cech cohomology sequence. The homomorphisms $\pi, \eta, \delta$ form a cohomology sequence

$$
\begin{aligned}
\cdots & \stackrel{\delta}{\rightarrow} H^{n}\left(X \bmod X_{0}, G\right) \stackrel{\pi}{\rightarrow} H^{n}(X, G) \stackrel{\eta}{\rightarrow} H^{n}\left(X_{0}, G\right) \\
& \stackrel{\delta}{\rightarrow} H^{n+1}\left(X \bmod X_{0}, G\right) \stackrel{\pi}{\rightarrow} \cdots
\end{aligned}
$$

which is exact in the sense that the kernel of each homomorphism coincides with the image of the preceding.

Now let $X^{\prime}$ be another topological space, $X_{0}^{\prime}$ a closed set of $X^{\prime}$, and $X_{*}^{\prime}=X^{\prime}-X_{0}^{\prime}$ its open complement. Let $f: X^{\prime} \rightarrow X$ be a given mapping with $f\left(X_{0}^{\prime}\right) \subset X_{0} . f$ induces homomorphisms of the cohomology groups $H^{n}(X, G)$, $H^{n}\left(X_{0}, G\right), H^{n}\left(X \bmod X_{0}, G\right)$ into $H^{n}\left(X^{\prime}, G\right), H^{n}\left(X_{0}^{\prime}, G\right), H^{n}\left(X^{\prime} \bmod X_{0}^{\prime}, G\right)$ respectively. All of these homomorphisms will be denoted simply by $f^{*}$ and described as follows.

Let $\xi \in H^{n}\left(X \bmod X_{0}, G\right)$ be an arbitrary element represented by a cocycle $z^{n}$ in $A_{*}$ for some covering $\alpha=\left\{a_{1}, a_{2}, \cdots, a_{r}\right\}$ of $X$. Let $\alpha^{\prime}=\left\{a_{1}^{\prime}, a_{2}^{\prime}, \cdots, a_{r}^{\prime}\right\}$ denote the covering of $X^{\prime}$ which consists of the open sets

$$
a_{i}{ }^{\prime}=f^{-1}\left(a_{i}\right) \quad(i=1,2, \cdots, r) .
$$

The correspondence $a_{i}^{\prime} \rightarrow a_{i}(i=1,2, \cdots, r)$ defines a simplicial mapping $t: A^{\prime} \rightarrow A$ such that $t\left(A_{0}^{\prime}\right) \subset A_{0} . t$ induces a homomorphism $t^{*}$ of the cocycles of $A_{*}$ into those of $A_{*}^{\prime}$. Then $f^{*}(\xi) \in H^{n}\left(X^{\prime} \bmod X_{0}^{\prime}, G\right)$ is the element represented by the cocycle $t^{*}\left(z^{n}\right)$ of $A_{*}^{\prime}$. The homomorphism $f^{*}$ for the groups $H^{n}(X, G)$ and $H^{n}\left(X_{0}, G\right)$ is similarly defined.

(1.2) Induced homomorphism. The homomorphisms $f^{*}$ of the cohomology groups of $X, X_{0}, X \bmod X_{0}$ into those of $X^{\prime}, X_{0}^{\prime}, X^{\prime} \bmod X_{0}^{\prime}$, induced by the mapping $f: X^{\prime} \rightarrow X$ with $f\left(X_{0}^{\prime}\right) \subset X_{0}$, form a homomorphism of the cohomology sequence in the sense that the following conditions of commutativity are satisfied:

$$
f^{*} \pi=\pi^{\prime} f^{*}
$$

$$
f^{*} \eta=\eta^{\prime} f^{*} \text {, }
$$




$$
f^{*} \delta=\delta^{\prime} f^{*}
$$

where $\pi^{\prime}, \eta^{\prime}, \delta^{\prime}$ denote the corresponding homomorphisms in the cohomology sequence of the dissection $\left(X_{0}^{\prime}, X_{*}^{\prime}\right)$. Further, if $g: X^{\prime \prime} \rightarrow X^{\prime}$ with $g\left(X_{0}^{\prime \prime}\right) \subset X_{0}^{\prime}$, then we have $(f g)^{*}=g^{*} f^{*}$.

2. Fundamental bridge theorems. Hereafter, we shall assume that (1) $X$ be a normal space and $X_{0}$ a closed set of $X$, (2) $Y$ be a connected ANR (and hence arcwise connected), (3) either $X_{0}$ or $Y$ be compact. Denote by $X_{*}=X-X_{0}$ the open complement. We shall use the typical notations of the foregoing section and understand that the nerves of the coverings are all geometrically realized. Without danger of ambiguity we may denote the vertices of the geometric nerve $A$ of a covering $\alpha=\left\{a_{1}, a_{2}, \cdots, a_{r}\right\}$ still by the symbols $a_{1}, a_{2}, \cdots, a_{r}$. The simplicial mappings of a finite geometric complex into another can also be considered as mappings in the set-theoretic meaning, linear on each simplex; and we shall so understand in what follows.

Let $\alpha$ be an arbitrary covering of $X$. A mapping $\phi_{\alpha}: X \rightarrow A$ is called a canonical mapping of $\alpha$, if for each point $x \in X, \phi_{\alpha}(x)$ is contained in the closure of the simplex $a_{i_{0}} a_{i_{1}} \cdots a_{i_{n}}$ of $A$, where $a_{i_{0}}, a_{i_{1}}, \cdots, a_{i_{n}}$ denote the members of $\alpha$ containing $x[15$, p. 40]. According to the fundamental mapping theorem of Lefschetz [15, p. 41], every covering $\alpha$ of $X$ has a canonical mapping $\left[15\right.$, p. 45]. Clearly $\phi_{\alpha}\left(X_{0}\right) \subset A_{0}$. It is also trivial that the canonical mappings of a given covering $\alpha$ of $X$ are homotopic to each other.

Let $f: X_{0} \rightarrow Y$ be a given mapping and $\alpha$ a covering of $X$. A mapping $\psi_{\alpha}: A_{0} \rightarrow Y$ is called a bridge mapping for $f$, if the partial mapping $\psi_{\alpha} \phi_{\alpha} \mid X_{0}$ is homotopic with $f$ for each canonical mapping $\phi_{\alpha}: X \rightarrow A$ of the covering $\alpha$. If such a bridge mapping $\psi_{\alpha}$ exists, $\alpha$ is said to be a bridge for the mapping $f$.

(2.1) Bridge Refinement theorem. For a given mapping $f: X_{0} \rightarrow Y$, any refinement $\beta$ of a bridge $\alpha$ is a bridge.

Proof. Let $\psi_{\alpha}: A_{0} \rightarrow Y$ be a bridge mapping for $f$; and $\phi_{\alpha}: X \rightarrow A, \phi_{\beta}: X \rightarrow B$ be arbitrary canonical mappings. Choose a simplicial projection $p_{\beta \alpha}: B \rightarrow A$. It remains to prove that $\psi_{\beta}=\psi_{\alpha} p_{\beta \alpha} \mid B_{0}$ is a bridge mapping for $f$; that is, $\psi_{\alpha} p_{\beta \alpha} \phi_{\beta} \mid X_{0}$ is homotopic with $f$. It is obvious that both $\phi_{\alpha}(x)$ and $p_{\beta \alpha} \phi_{\beta}(x)$ are contained in the closure of the simplex $a_{i_{0}} a_{i_{1}} \cdots a_{i_{n}}$ of $A$, where $a_{i_{0}}$, $a_{i_{1}}, \cdots, a_{i_{n}}$ denote the members of $\alpha$ containing $x$. Hence $\phi_{\alpha}$ and $p_{\beta \alpha} \phi_{\beta}$ are homotopic; and our theorem follows from the relation

$$
f \simeq \psi_{\alpha} \phi_{\alpha}\left|X_{0} \simeq \psi_{\alpha} \not_{\beta \alpha} \phi_{\beta}\right| X_{0}
$$

(2.2) BRIDGE EXISTENCE THEOREM. Every mapping $f: X_{0} \rightarrow Y$ has a bridge $\alpha$.

Proof. According to a theorem of Wojdyslawski [18, p. 186], $Y$ can be imbedded as a closed set of a convex subset $Z$ of a separable Banach space 
$W[2$, p. 53]. Since $Y$ is an ANR, there exist an open set $V$ of $Z$ containing $Y$ and a retraction $\theta: V \rightarrow Y[14$, p. 40]. For each point $y \in Y$, let $S(y)$ denote the greatest open spherical neighborhood of $y$ in $Z$ such that $S(y) \subset V$. Let us denote the distance function of $W$ by $\rho$; then for each positive number $\epsilon, y_{i} \in Y(i=1,2)$ and $\rho\left(y_{1}, y_{2}\right)<\epsilon$ implies that the radius of $S\left(y_{2}\right)$ is at least equal to that of $S\left(y_{1}\right)$ diminished by $\epsilon$.

Now let $f: X_{0} \rightarrow Y$ be a given mapping. We are going to prove the existence of a covering $\alpha_{0}^{*}=\left\{a_{1}^{*}, a_{2}{ }^{*}, \cdots, a_{r}^{*}\right\}$ of $X_{0}$ such that for each $a_{i}{ }^{*} \in \alpha_{0}{ }^{*}$ $(i=1,2, \cdots, r)$ there is a corresponding point $y_{i} \in Y$ with $f\left(a_{i}^{*}\right) \subset S\left(y_{i}\right)$. First, let us assume the compactness of $Y$. Then there exist a finite number of points $y_{1}, y_{2}, \cdots, y_{r}$ such that the open sets $S\left(y_{i}\right)(i=1,2, \cdots, r)$ cover $Y$. Let $a_{i}^{*}=f^{-1}\left(S\left(y_{i}\right) \cap Y\right)$, then $a_{0}^{*}=\left\{a_{1}^{*}, a_{2}^{*}, \cdots, a_{r}^{*}\right\}$ is a covering of $X_{0}$ of the kind required. Second, let us assume the compactness of $X_{0}$. The inverse images $f^{-1}(S(y) \cap Y), y \in Y$, cover $X_{0}$. The compactness of $X_{0}$ implies the existence of a finite number of these, say $f^{-1}\left(S\left(y_{i}\right) \cap Y\right)(i=1,2, \cdots, r)$, already cover $X_{0}$. Set $a_{i}^{*}=f^{-1}\left(S\left(y_{i}\right) \cap Y\right)$; it follows that the covering $\alpha_{0}^{*}$ $=\left\{a_{1}^{*}, a_{2}{ }^{*}, \cdots, a_{r}^{*}\right\}$ of $X_{0}$ is of the kind required. This proves our assertion.

Having given a covering $\alpha_{0}^{*}=\left\{a_{1}^{*}, a_{2}{ }^{*}, \cdots, a_{r}^{*}\right\}$ described as above, we denote for simplicity $S_{i}=S\left(y_{i}\right)$. For each $a_{i}^{*} \in \alpha_{0}^{*}$, choose an open set $a_{i}$ of $X$ such that $a_{i} \cap X_{0}=a_{i}^{*}$. Set $a_{r+1}=X-X_{0}$. Then $\alpha=\left\{a_{1}, a_{2}, \cdots, a_{r}, a_{r+1}\right\}$ is a covering of $X$ with $\alpha_{0}=\alpha_{0}^{*}$. We shall complete our proof by showing that $\alpha$ is a bridge for $f$.

We are going to construct a bridge mapping $\psi_{\alpha}: A_{0} \rightarrow Y$. The vertices of $A_{0}$ are also denoted by $a_{1}, a_{2}, \cdots, a_{r}$. Let $Q$ denote the first barycentric subdivision of $A_{0}$; then a vertex $v$ of $Q$ is the centroid of some simplex $a_{i_{0}} a_{i_{1}} \cdots a_{i_{n}}$ of $A_{0}$. Since $f\left(a_{i \mu}^{*}\right) \subset S_{i_{\mu}}(\mu=0,1, \cdots, n)$ and the intersection of the sets $a_{i_{\mu}}^{*}(\mu=0,1, \cdots, n)$ is nonempty, the spherical neighborhoods $S_{i_{\mu}}$ $(\mu=0,1, \cdots, n)$ have a nonvoid intersection. In this intersection, let us choose a point $g(v)$. Since $Z$ is a convex subset of a Banach space $W$, we can extend $g$ to be a mapping $g: A_{0} \rightarrow Z$ which is linear on each simplex of $Q$. Let $\sigma=v_{0} v_{1} \cdots v_{q}$ be an arbitrary simplex of $Q$, then it follows from the construction of $g$ that there exists some $S_{i}$ containing all the points $g\left(v_{\mu}\right)$ $(\mu=0,1, \cdots, q)$. Since $S_{i}$ is a spherical neighborhood in a convex set $Z$, we have that $g(\sigma) \subset S_{i}$. Hence $g\left(A_{0}\right) \subset V$. Define a mapping $\psi_{\alpha}: A_{0} \rightarrow Y$ by taking $\psi_{\alpha}=\theta g$. It remains to prove that $\psi_{\alpha}$ is a bridge mapping for $f$.

Let $x \in X_{0}$ be an arbitrary point and denote by $a_{i_{0}}^{*}, a_{i_{1}}^{*}, \cdots, a_{i_{n}}^{*}$ the members of the covering $\alpha_{0}^{*}$ containing $x$. Then $f(x) \in S_{i_{\mu}} \cap Y(\mu=0,1, \cdots, n)$. Let $\phi_{\alpha}: X \rightarrow A$ be an arbitrary canonical mapping of $\alpha$; then $\phi_{\alpha}(x)$ is a point of the closure of the simplex $a_{i_{0}} a_{i_{1}} \cdots a_{i_{n}}$ of $A_{0}$. The existence of some $S_{i_{\mu}}$ $(0 \leqq \mu \leqq n)$ which contains $g \phi_{\alpha}(x)$ follows. Let $\xi_{t}(x)$ denote the point which divides the line-segment joining $f(x)$ to $g \phi_{\alpha}(x)$ in the ratio $t:(1-t)$ for each $0 \leqq$ $t \leqq 1$. Since both $f(x)$ and $g \phi_{\alpha}(x)$ are points of $S_{i_{\mu}}$, we have $\xi_{t}(x) \in S_{i}(0 \leqq t \leqq 1)$. Define a homotopy $f_{t}: X_{0} \rightarrow Y(0 \leqq t \leqq 1)$ by taking 


$$
f_{t}(x)=\theta \xi_{t}(x)
$$$$
\left(x \in X_{0}, 0 \leqq t \leqq 1\right) ;
$$

then we have $f_{0}=f$ and $f_{1}=\psi_{\alpha} \phi_{\alpha} \mid X_{0}$. Hence $\psi_{\alpha}$ is a bridge mapping for $f$ and our theorem is proved. Q.E.D.

The following corollary is an immediate consequence of (2.1) and (2.2).

(2.3) The set $\mathfrak{B}_{f}$ of all bridges for a given mapping $f: X_{0} \rightarrow Y$ is residual $[16$, p. 10] (and hence cofinal) in the directed set $\subseteq$ of all coverings of $X$.

(2.4) BRIDGe HOMOTOPY THEOREM. If $\alpha, \beta$ be two bridges for a given mapping $f: X_{0} \rightarrow Y$, where $X_{0}$ is compact, and if $\psi_{\alpha}: A_{0} \rightarrow Y, \psi_{\beta}: B_{0} \rightarrow Y$ be bridge mappings; then there exists a common refinement $\gamma$ of $\alpha$ and $\beta$ such that $\psi_{\alpha} p_{\gamma \alpha} \mid C_{0}$ and $\psi_{\beta} p_{\gamma \beta} \mid C_{0}$ are homotopic, where $p_{\gamma \alpha}: C \rightarrow A, p_{\gamma \beta}: C \rightarrow B$ are arbitrary simplicial projections.

Proof. We shall use the notations in the proof of the bridge existence theorem. From the definition of the spherical neighborhoods $S(y)$ follows quite obviously the existence of a sufficiently fine simplicial subdivision $A_{0}^{\prime}$ of $A_{0}$ such that for each vertex $a_{i}^{\prime}$ of $A_{0}^{\prime}$ we have $\psi_{\alpha} \mathrm{Cl}\left(\mathrm{St} a_{i}^{\prime \prime}\right) \subset S\left(\psi_{\alpha}(x)\right)$, where $x$ is an arbitrary point of the closure $\mathrm{Cl}\left(\mathrm{St} a_{i}^{\prime}\right)$ of the star St $a_{i}^{\prime}$ in $A_{0}^{\prime}$. Let $A^{\prime}$ be a simplicial subdivision of $A$ which coincides with $A_{0}^{\prime}$ on $A_{0}$. Let $B^{\prime}$ be a simplicial subdivision of $B$ defined similarly for the mapping $\psi_{\beta}$. The totality of the open sets $\phi_{\alpha}^{-1}$ (St $\left.a_{i}^{\prime}\right)$, where the $a_{i}^{\prime}$ are vertices of $A^{\prime}$ and $\phi_{\alpha}$ is a canonical mapping of $\alpha$, form a covering $\alpha^{*}$ of $X$ which refines $\alpha$. Similarly, the covering $\beta^{*}=\left\{\phi_{\beta}^{-1}\left(\mathrm{St} b_{i}^{\prime}\right)\right\}$ of $X$ refines $\beta$.

By the definition of bridge mappings, $\psi_{\alpha} \phi_{\alpha} \mid X_{0}$ and $\psi_{\beta} \phi_{\beta} \mid X_{0}$ are both homotopic with $f$; hence there exists a mapping $F: X_{0} \times I \rightarrow Y$, where $I$ denotes the closed interval $(0,1)$ such that

$$
F(x, 0)=\psi_{\alpha} \phi_{\alpha}(x), \quad F(x, 1)=\psi_{\beta} \phi_{\beta}(x) \quad\left(x \in X_{0}\right) .
$$

From the continuity of $F$ and the definition of $S(y)$, it follows that for each $x \in X_{0}$ and each $t \in I$ there exist an open set $U(x)$ of $X_{0}$ containing $x$ and an open set $T(t)$ of $I$ (which may be assumed to be an interval) containing $t$ such that

$$
F(U(x) \times T(t)) \subset S(F(\zeta))
$$

where $\zeta$ is an arbitrary point of $U(x) \times T(t)$. Since $X_{0} \times I$ is compact, there exist a finite number of points $\left(x_{i}, t_{i}\right)(i=1,2, \cdots, p)$ such that the open sets $\left\{U\left(x_{i}\right) \times T\left(t_{i}\right)\right\}(i=1,2, \cdots, p)$ cover $X_{0} \times I$. For each $x \in X_{0}$, let $M(x)$ denote the intersection of the members of the collection $\left\{U\left(x_{i}\right)\right\}$ $(i=1,2, \cdots, p)$ which contain $x$. Since the collection $\left\{U\left(x_{i}\right)\right\}$ is finite, $M(x)$ is an open set. The collection $\{M(x)\}\left(x \in X_{0}\right)$ has a finite subcollection $\delta_{0}=\left\{d_{1}, d_{2}, \cdots, d_{q}\right\}$ which covers $X_{0}$. For each $d_{i}(i=1,2, \cdots, q)$ choose an open set $d_{i}^{*}$ of $X$ with $d_{i}=d_{i}^{*} \cap X_{0}$, and let $d_{q+1}^{*}=X-X_{0}$. Then $\delta^{*}=\left\{d_{1}^{*}, d_{2}^{*}\right.$, $\left.\cdots, d_{q}^{*}, d_{q+1}^{*}\right\}$ is a covering of $X$ with $\delta_{0}^{*}=\delta_{0}$. 
Let $\gamma=\left\{c_{1}, c_{2}, \cdots, c_{i}\right\}$ be a common refinement of the coverings $\alpha^{*}, \beta^{*}$, and $\delta^{*} ;$ further let

$$
p_{\gamma \alpha}: C \rightarrow A, \quad p_{\gamma \beta}: C \rightarrow B
$$

be arbitrary simplicial projections. We are going to prove that $\psi_{\alpha} p_{\gamma \alpha} \mid C_{0}$ and $\psi_{\beta} p_{\gamma \beta} \mid C_{0}$ are homotopic.

For each $t \in I$, the intersection of the members of $\left\{T\left(t_{i}\right)\right\}(i=1,2, \cdots, p)$ containing $t$ is an open set; choose a closed interval $J(t)$ containing $t$ in its interior and contained in this intersection. There are a finite number of such closed intervals $J_{1}, J_{2}, \cdots, J_{k}$ which cover $I$. Let

$$
0=\tau_{0}<\tau_{1}<\cdots<\tau_{s-1}<\tau_{s}=1
$$

be the set of end points of these intervals $J_{1}, J_{2}, \cdots, J_{k}$. Then it is easily seen that for each $c_{i} \in \gamma$ and each closed interval $\left(\tau_{\iota-1}, \tau_{\iota}\right)(\iota=1,2, \cdots, s)$ we have $F\left(\left(c_{i} \cap X_{0}\right) \times\left(\tau_{\iota-1}, \tau_{\imath}\right)\right) \subset S(F(\zeta)), \zeta$ being an arbitrary point of $\left(c_{i} \cap X_{0}\right) \times\left(\tau_{\iota-1}, \tau_{\iota}\right)$.

By the constructions of the coverings $\alpha^{*}$ and $\beta^{*}$, their nerves $A^{*}$ and $B^{*}$ can be identified with subcomplexes of $A^{\prime}$ and $B^{\prime}$ respectively by the correspondences $a_{i}^{*} \rightarrow a_{i}^{\prime}$ and $b_{i}^{*} \rightarrow b_{i}^{\prime}$.

Take arbitrary simplicial projections

$$
p_{\gamma \alpha}^{*}: C \rightarrow A^{*} \subset A^{\prime}, \quad p_{\gamma \beta}^{*}: C \rightarrow B^{*} \subset B^{\prime}
$$

and define a mapping $G: C_{0} \times I \rightarrow Z$ described as follows.

For each point $c \in C_{0}$, let us define

$$
G(c, 0)=\psi_{\alpha} p_{\gamma \alpha}^{*}(c), \quad G(c, 1)=\psi_{\beta} p_{\gamma \beta}^{*}(c) .
$$

If $\sigma=c_{i_{0}} c_{i_{1}} \cdots c_{i_{n}}$ be an arbitrary simplex of $C_{0}$ and $x$ be an arbitrary point of the intersection

$$
L=c_{i 0} \cap c_{i 1} \cap \cdots \cap c_{i n} \cap X_{0},
$$

then it is not difficult to see the following inclusions:

$$
G(\sigma \times 0) \subset S(F(x, 0)), \quad G(\sigma \times 1) \subset S(F(x, 1)) .
$$

Next, for each vertex $c_{i} \in C_{0}$, choose a fixed point $x_{i} \in c_{i} \cap X_{0}$. For each vertex $c_{i} \in C_{0}$ and each $\tau_{\iota}(\iota=1,2, \cdots, s-1)$, define $G\left(c_{i}, \tau_{\iota}\right)=F\left(x_{i}, \tau_{\iota}\right)$. If $\sigma=c_{i_{0}} c_{i_{1}} \cdots c_{i_{n}}$ be an arbitrary simplex of $C_{0}$ and $x$ be an arbitrary point of $L=c_{i_{0}} \cap c_{i_{1}} \cap \cdots \cap c_{i_{n}} \cap X_{0}$, then we have

$$
G\left(c_{i_{\mu}}, \tau_{\iota}\right) \subset S\left(F\left(x, \tau_{\iota-1}\right)\right) \cap S\left(F\left(x, \tau_{\iota}\right)\right) \cap S\left(F\left(x, \tau_{\iota+1}\right)\right)
$$

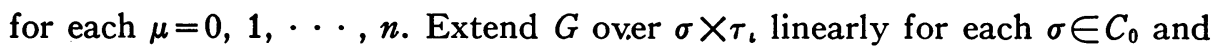


each $\tau_{\iota}(\iota=1,2, \cdots, s-1)$. Then we have

$$
G\left(\sigma \times \tau_{\iota}\right) \subset S\left(F\left(x, \tau_{\iota-1}\right)\right) \cap S\left(F\left(x, \tau_{\iota}\right)\right) \cap S\left(F\left(x, \tau_{\iota+1}\right)\right) .
$$

Since, for each point $c \in \sigma$, both $G\left(c, \tau_{\iota-1}\right)$ and $G\left(c, \tau_{\iota}\right)(\iota=1,2, \cdots, s)$ are contained in $S\left(F\left(x, \tau_{\iota}\right)\right)$, one can extend $G$ linearly on each $c \times\left(\tau_{\iota-1}, \tau_{\iota}\right)$. This completes the construction of the mapping $G: C_{0} \times I \rightarrow Z$. It is trivial that $G\left(C_{0} \times I\right) \subset V$.

Define a homotopy $g_{t}: C_{0} \rightarrow Y(0 \leqq t \leqq 1)$ by taking $g_{t}(c)=\theta G(c, t)\left(c \in C_{0}\right.$, $0 \leqq t \leqq 1)$; then we have $g_{0}=\psi_{\alpha} p_{\gamma \alpha}^{*}$ and $g_{1}=\psi_{\beta} p_{\gamma \beta}^{*}$. Since it is obvious that $p_{\gamma \alpha}^{*} \simeq p_{\gamma \alpha}$ and $p_{\gamma \beta}^{*} \simeq p_{\gamma \beta}$, we have

$$
\psi_{\alpha} p_{\gamma \alpha}\left|C_{0} \simeq \psi_{\alpha} p_{\gamma \alpha}^{*}\right| C_{0} \simeq \psi_{\beta} p_{\gamma \beta}^{*}\left|C_{0} \simeq \psi_{\beta} p_{\gamma \beta}\right| C_{0}
$$

hence our theorem follows. Q.E.D.

\section{THE GENERAL THEORY}

3. $n$-extensibility. We shall denote by $A^{n}$ the $n$-skeleton of a complex $A$, that is, the totality of the simplexes of $A$ with dimensions not exceeding $n$.

A mapping $f: X_{0} \rightarrow Y$ is said to be $n$-extensible, if $f$ has a bridge $\alpha$ with a bridge mapping $\psi_{\alpha}: A_{0} \rightarrow Y$ which has an extension $\psi_{\alpha}^{\prime}: A_{0} \cup A^{n} \rightarrow Y$. Since $Y$ is arcwise connected, the following statement is trivial.

(3.1) Every mapping $f: X_{0} \rightarrow Y$ is 1-extensible.

For a given mapping $f: X_{0} \rightarrow Y$, the least upper bound of the set of integers $n$ such that $f$ is $n$-extensible is called the extension index of the mapping $f$. The following statement is obvious from the very definition.

(3.2) Homotopic mappings have the same extension index.

Some set-theoretic meaning of the $n$-extensibility is shown by the following theorem.

(3.3) If $X$ be metric separable with $\operatorname{dim}\left(X-X_{0}\right) \leqq m$ and if $f: X_{0} \rightarrow Y$ be $n$-extensible, then there exists a closed set $X_{1}$ of $X$ contained in $X-X_{0}$ such that $\operatorname{dim} X_{1}<m-n$ and $f$ has an extension $f^{\prime}: X-X_{1} \rightarrow Y$.

Proof. By the definition of $n$-extensibility, there exist a bridge $\alpha$ and a bridge mapping $\psi_{\alpha}: A_{0} \rightarrow Y$ for $f$, which has an extension $\psi_{\alpha}^{\prime}: A_{0} \cup A^{n} \rightarrow Y$. Let $\phi: X \rightarrow A$ be an arbitrary mapping. We shall prove an assertion that there exist a closed set $X_{1}$ of $X$ contained in $X-X_{0}$ with $\operatorname{dim} X_{1}<m-n$ and $a$ homotopy $h_{t}: X-X_{1} \rightarrow A \quad(0 \leqq t \leqq 1)$ such that $h_{0}=\phi \mid X-X_{1}, h_{1}\left(X-X_{1}\right) \subset A_{0}$ $\cup A^{n}$, and $h_{t}(x)=\phi(x)$ for each $x \in \phi^{-1}\left(A_{0} \cup A^{n}\right)$ and each $0 \leqq t \leqq 1$.

Our assertion is trivial if $\operatorname{dim} A \leqq n$. Assume the assertion for $\operatorname{dim} A \leqq p-1$, and now suppose $\operatorname{dim} A=p>n$. Let $\sigma_{1}, \sigma_{2}, \cdots, \sigma_{q}$ be the $p$-simplexes of $A-A_{0}$, and denote by $\bar{\sigma}_{i}, \partial \sigma_{i}$ respectively the closure and the boundary of the open simplex $\sigma_{i}$. Let

$$
F_{i}=\phi^{-1}\left(\bar{\sigma}_{i}\right), \quad B_{i}=\phi^{-1}\left(\partial \sigma_{i}\right) \quad(i=1,2, \cdots q) .
$$


$F_{i}, B_{i}$ are closed sets of $X$ and $F_{i}-B_{i} \subset X-X_{0}$. Hence we have $\operatorname{dim}\left(F_{i}-B_{i}\right)$ $\leqq m$. It follows from the duality theorem of Eilenberg-Borsuk $[4$, p. 162] that there is a closed subset $H_{i}$ of $F_{i}$ contained in $F_{i}-B_{i}$ and with

$$
\operatorname{dim} H_{i} \leqq m-(p-1)-1<m-n
$$

such that the partial mapping $\phi \mid B_{i}$ has an extension $\theta_{i}: F_{i}-H_{i} \rightarrow \partial \sigma_{i}$. Let $H$ denote the union of all the subsets $H_{i}(i=1,2, \cdots, q)$, then $H$ is a closed set of $X$ which is contained in $X-X_{0}$ and with $\operatorname{dim} H<m-n$. Define a mapping $\theta: X-H \rightarrow A_{0} \cup A^{p-1}$ by taking

$$
\theta(x)=\left\{\begin{array}{lr}
\theta_{i}(x) & \left(x \in F_{i}-H_{i}\right), \\
\phi(x) & \left(x \in X-U F_{i}\right) .
\end{array}\right.
$$

For each $x \in F_{i}-H_{i}$, both $\phi(x)$ and $\theta(x)$ are contained in $\bar{\sigma}_{i}$. Define a homotopy $\xi_{t}: X-H \rightarrow A(0 \leqq t \leqq 1)$ as follows: If $x$ belongs to some $F_{i}-H_{i}$, let $\xi_{t}(x)$ be the point which divides the line-segment joining $\phi(x)$ to $\theta(x)$ in the ratio $t:(1-t)$; otherwise, let $\xi_{t}(x)=\phi(x)$. Then we have $\xi_{0}=\phi \mid X-H$, $\xi_{1}=\theta$ and $\xi_{t}(x)=\phi(x)$ for each $x \in \phi^{-1}\left(A_{0} \cup A^{p-1}\right)$ and each $0 \leqq t \leqq 1$. From our assumption of the induction follows the existence of a closed subset $J$ of $X-H$ contained in $X-\left(H \cup X_{0}\right)$ and with $\operatorname{dim} J<m-n$ and a homotopy

$$
\eta_{t}: X-H-J \rightarrow A_{0} \cup A^{p-1} \quad(0 \leqq t \leqq 1)
$$

such that $\eta_{0}=\theta \mid X-H-J, \eta_{1}(X-H-J) \subset A_{0} \cup A^{n}$, and that $\eta_{t}(x)=\theta(x)$ for each $x \in \theta^{-1}\left(A_{0} \cup A^{n}\right)$ and each $0 \leqq t \leqq 1$. Let $X_{1}=H \cup J$. Since $J$ is closed in $X-H$ and $H$ in $X, X_{1}$ is a closed set of $X$ contained in $X-X_{0}$. Since $H$ is closed, it follows from the sum theorem of dimension theory [13, p. 32] that $\operatorname{dim} X_{1}<m-n$. Define a homotopy $h_{t}: X-X_{1} \rightarrow A(0 \leqq t \leqq 1)$ by taking

$$
h_{t}(x)= \begin{cases}\xi_{2 t}(x) & \left(x \in X-X_{1}, 0 \leqq t \leqq 1 / 2\right), \\ \eta_{2 t-1}(x) & \left(x \in X-X_{1}, 1 / 2 \leqq t \leqq 1\right) ;\end{cases}
$$

then $h_{0}=\phi \mid X-X_{1}, h_{1}\left(X-X_{1}\right) \subset A_{0} \cup A^{n}$, and $h_{t}(x)=\phi(x)$ for each $0 \leqq t \leqq 1$ and each $x \in \phi^{-1}\left(A_{0} \cup A^{n}\right)$. Hence our assertion is proved.

Now let $\phi=\phi_{\alpha}$ be a canonical mapping of $\alpha$; then $\psi_{\alpha}^{\prime} h_{1}: X-X_{1} \rightarrow Y$ is an extension of $\psi_{\alpha} \phi_{\alpha} \mid X_{0}$. It follows from Borsuk's theorem [13, p. 86] that $f$ has an extension $f^{\prime}: X-X_{1} \rightarrow Y$. This completes our proof. Q.E.D.

4. Obstruction sets. In the present section, we shall assume an additional condition that $Y$ be $n$-simple in the sense of Eilenberg [8]. Let $\pi^{n}=\pi^{n}(Y)$ denote the $n$th homotopy group of $Y$.

Let $f: X_{0} \rightarrow Y$ be a given mapping. We are going to define the $(n+1)$ dimensional obstruction set $\Omega^{n+1}(f) \subset H^{n+1}\left(X \bmod X_{0}, \pi^{n}\right)$ of the mapping $f$ as follows.

If $f$ is not $n$-extensible, we define $\Omega^{n+1}(f)$ to be the vacuous set. Now suppose $f$ to be $n$-extensible. Then there exist a bridge $\alpha$ and a bridge mapping 
$\psi_{\alpha}: A_{0} \rightarrow Y$ which has an extension $\psi_{\alpha}^{\prime}: A_{0} \cup A^{n} \rightarrow Y$. For each $(n+1)$-simplex $\sigma^{n+1} \in A$ the partial mapping $\psi_{\alpha}^{\prime} \mid \partial \sigma^{n+1}$, the $n$-sphere $\partial \sigma^{n+1}$ being oriented by the $n$-cycle $\partial \sigma^{n+1}$, defines uniquely an element $\left(\psi_{\alpha}^{\prime}, \sigma^{n+1}\right)$ of the group $\pi^{n}$. Then the $(n+1)$-chain

$$
w^{n+1}\left(\psi_{\alpha}^{\prime}\right)=\sum_{i}\left(\psi_{\alpha}^{\prime}, \sigma_{i}^{n+1}\right) \sigma_{i}^{n+1}
$$

is a cocycle in $A-A_{0}\left[9\right.$, p. 237]; hence it represents an element $\omega^{n+1}\left(\psi_{\alpha}^{\prime}\right)$ of the group $H^{n+1}\left(X \bmod X_{0}, \pi^{n}\right)$, called an obstruction element of $f . \Omega^{n+1}(f)$ is defined to be the set of all obstruction elements of $f$ in the group $H^{n+1}\left(X \bmod X_{0}, \pi^{n}\right)$.

The following statements are trivial.

(4.1) $A$ mapping $f: X_{0} \rightarrow Y$ is n-extensible if and only if $\Omega^{n+1}(f)$ is nonempty.

(4.2) Homotopic mappings have the same $(n+1)$-dimensional obstruction set.

(4.3) Fundamental extension lemma. $A$ mapping $f: X_{0} \rightarrow Y$ is $(n+1)$ extensible if and only if $\Omega^{n+1}(f)$ contains the zero element of $H^{n+1}(X \bmod$ $\left.X_{0}, \pi^{n}\right)$.

Proof. Necessity. Suppose $f: X_{0} \rightarrow Y$ to be $(n+1)$-extensible; then there exist a bridge $\alpha$ and a bridge mapping $\psi_{\alpha}: A_{0} \rightarrow Y$ which has an extension $\psi_{\alpha}^{\prime}: A_{0} \cup A^{n+1} \rightarrow Y$. Since $\psi_{\alpha}^{\prime}$ is defined over $\sigma_{i}^{n+1},\left(\psi_{\alpha}^{\prime}, \sigma_{i}^{n+1}\right)$ is the zero element of $\pi^{n}$. Hence $w^{n+1}\left(\psi_{\alpha}^{\prime}\right)=0$ and $\Omega^{n+1}(f)$ contains the zero element of $H^{n+1}\left(X \bmod X_{0}, \pi^{n}\right)$.

Sufficiency. Suppose that $\Omega^{n+1}(f)$ contains the zero element of $H^{n+1}\left(X \bmod X_{0}, \pi^{n}\right)$. Then there exist a bridge $\alpha$ and a bridge mapping $\psi_{\alpha}: A_{0} \rightarrow Y$ which has an extension $\psi_{\alpha}^{\prime}: A_{0} \cup A^{n} \rightarrow Y$ such that the cocycle $w^{n+1}\left(\psi_{\alpha}^{\prime}\right)$ represents the zero element of $H^{n+1}\left(X \bmod X_{0}, \pi^{\dot{n}}\right)$. According to the definition of Cech cohomology groups (\$1), there exists a refinement $\beta$ of $\alpha$ such that the cocycle $p_{\beta \alpha}^{*}\left(w^{n+1}\left(\psi_{\alpha}^{\prime}\right)\right)$ in $B-B_{0}$ induced by a simplicial projection $p_{\beta \alpha}: B \rightarrow A$ is cohomologous to zero in $B-B_{0}$. Let $\psi_{\beta}=\psi_{\alpha} p_{\beta \alpha} \mid B_{0}$. One can see from the proof of (2.1) that $\psi_{\beta}$ is a bridge mapping for $f$. $\psi_{\beta}$ has an extension $\psi_{\beta}^{\prime}: B_{0} \cup B^{n} \rightarrow Y$ given by $\psi_{\beta}^{\prime}=\psi_{\alpha}^{\prime} p_{\beta \alpha} \mid B_{0} \cup B^{n}$. It is quite obvious that

$$
w^{n+1}\left(\psi_{\beta}^{\prime}\right)=p_{\beta \alpha}^{*}\left(w^{n+1}\left(\psi_{\alpha}^{\prime}\right)\right) \text {. }
$$

Since $w^{n+1}\left(\psi_{\beta}^{\prime}\right)$ cobounds in $B-B_{0}$, it follows from the first extension theorem of Eilenberg [9, p. 239], that there is mapping $\psi_{\beta}^{\prime \prime}: B_{0} \cup B^{n+1} \rightarrow Y$ such that $\psi_{\beta}^{\prime \prime}=\psi_{\beta}^{\prime}$ on $B_{0} \cup B^{n-1}$. Hence $f$ is $(n+1)$-extensible and our proof is completed. Q.E.D.

The following theorem is an immediate consequence of (4.3) and (4.1).

(4.4) If $Y$ is $r$-simple and $H^{r+1}\left(X \bmod X_{0}, \pi^{r}\right)=0$ for each $0<r<n$, then every mapping $f: X_{0} \rightarrow Y$ is n-extensible. 
Now suppose $X^{\prime}$ to be another normal space and $g: X^{\prime} \rightarrow X$ to be a given mapping. Let $\alpha=\left\{a_{1}, a_{2}, \cdots, a_{r}\right\}$ be an arbitrary covering of $X$ and $\alpha^{\prime}=\left\{a_{1}^{\prime}, a_{2}^{\prime}, \cdots, a_{r}^{\prime}\right\}$ be the covering of $X^{\prime}$ which consists of the open sets $a_{i}^{\prime}=g^{-1}\left(a_{i}\right)(i=1,2, \cdots, r)$. Denote by $A$ and $A^{\prime}$ the geometric nerves of $\alpha$ and $\alpha^{\prime}$. The correspondence $a_{i}^{\prime} \rightarrow a_{i}(i=1,2, \cdots, r)$ determines a unique simplicial mapping $t: A^{\prime} \rightarrow A$ Let $\phi: X \rightarrow A, \phi^{\prime}: X^{\prime} \rightarrow A^{\prime}$ be canonical mappings of $\alpha$ and $\alpha^{\prime}$ respectively.

(4.5) The mappings $\phi g$ and $t \phi^{\prime}$ of $X^{\prime}$ into $A$ are homotopic.

Proof. Let $x^{\prime}$ be an arbitrary point of $X^{\prime}$. Let $a_{i_{0}}^{\prime}, a_{i_{1}}^{\prime}, \cdots, a_{i_{n}}^{\prime}$ be the members of $\alpha^{\prime}$ containing $x^{\prime}$. Then $g\left(x^{\prime}\right) \in a_{i_{\mu}}$ for each $\mu=0,1, \cdots, n$. Hence both $\phi g\left(x^{\prime}\right)$ and $t \phi^{\prime}\left(x^{\prime}\right)$ are contained in the simplex $a_{i_{0}} a_{i_{1}} \cdots a_{i_{n}}$ of $A$; and the mappings $\phi g$ and $t \phi^{\prime}$ are then homotopic. Q.E.D.

Let $X_{0}^{\prime}$ be a closed set $X^{\prime}$ and $g: X^{\prime} \rightarrow X$ be a mapping such that $g\left(X_{0}^{\prime}\right)$ $\subset X_{0}$. Denote by $g^{*}: H^{n+1}\left(X \bmod X_{0}, \pi^{n}\right) \rightarrow H^{n+1}\left(X^{\prime} \bmod X_{0}^{\prime}, \pi^{n}\right)$ the homomorphism induced by $g(\$ 1)$.

(4.6) If $f: X_{0} \rightarrow Y$ is n-extensible, then so is the mapping $f^{\prime}=f g \mid X_{0}^{\prime}$ and $\Omega^{n+1}\left(f^{\prime}\right)$ contains the image $g^{*}\left(\Omega^{n+1}(f)\right)$ under the homomorphism $g^{*}$.

Proof. Let $\xi \in \Omega^{n+1}(f)$ be an arbitrary element. By definition, there exist a bridge $\alpha$ and a bridge mapping $\psi_{\alpha}: A_{0} \rightarrow Y$ which has an extension $\psi_{\alpha}$ : $A_{0} \cup A^{n} \rightarrow Y$ with the cocycle $w^{n+1}\left(\psi_{\alpha}^{\prime}\right)$ representing the element $\xi$. Let the members of $\alpha$ be the open sets $a_{1}, a_{2}, \cdots, a_{r}$; and denote by $\alpha^{\prime}=\left\{a_{1}^{\prime}, a_{2}^{\prime}\right.$, $\left.\cdots, a_{r}^{\prime}\right\}$ the covering of $X^{\prime}$ which consists of the open sets $\alpha_{i}^{\prime}=g^{-1}\left(a_{i}\right)$. Let $\phi: X \rightarrow A$ and $\phi^{\prime}: X^{\prime} \rightarrow A^{\prime}$ be canonical mappings, and $t: A^{\prime} \rightarrow A$ denote the simplicial mapping determined by the correspondence $a_{i}{ }^{\prime} \rightarrow a_{i}$. Since $\psi_{\alpha}$ is a bridge mapping for $f$, we have $\psi_{\alpha} \phi \mid X_{0} \sim f$. Hence, by (4.5),

$$
\psi_{\alpha} t \phi^{\prime}\left|X_{0}^{\prime} \simeq \psi_{\alpha} \phi g\right| X_{0}^{\prime} \simeq f g \mid X_{0}^{\prime}=f^{\prime} .
$$

Therefore, $\alpha^{\prime}$ is a bridge for $f^{\prime}$ with $\psi_{\alpha} t \mid A_{0}^{\prime}$ as a bridge mapping. Since $\psi_{\alpha} t \mid A_{0}^{\prime}$ has an extension $\psi_{\alpha}^{\prime} t \mid A_{0}^{\prime} \cup A^{\prime n}, f^{\prime}$ is $n$-extensible. Since it is obvious that

$$
\omega^{n+1}\left(\psi_{\alpha}^{\prime} t\right)=g^{*}\left(\omega^{n+1}\left(\psi_{\alpha}^{\prime}\right)\right),
$$

the inclusion $\Omega^{n+1}\left(f^{\prime}\right) \supset g^{*}\left(\Omega^{n+1}(f)\right)$ follows. Q.E.D.

5. General extension theorems. In the present section, let $m$ be a finite positive integer. If $X$ is metric separable, we assume $\operatorname{dim}\left(X-X_{0}\right) \leqq m$; otherwise, we assume $\left(^{3}\right) \operatorname{dim} \mathrm{Cl}\left(X-X_{0}\right) \leqq m$, where $\mathrm{Cl}\left(X-X_{0}\right)$ denotes the closure of $X-X_{0}$. As in $\$ 4$, denote by $\pi^{r}$ the $r$ th homotopy group of $Y$.

(5.1) If $Y$ is $r$-simple and $H^{r+1}\left(X \bmod X_{0}, \pi^{r}\right)=0$ for each $n \leqq r<m$, then every $n$-extensible mapping $f: X_{0} \rightarrow Y$ has an extension $f^{\prime}: X \rightarrow Y$.

Proof. By the recurrent applications of (4.1) and (4.3), one can see that $f$

(3) Further, we assume that $X_{0}$ has the homotopy extension property in $X$ with respect to $Y$; that is to say, Borsuk's theorem $[13 ;$ p. 86] holds. 
is $m$-extensible. Then there exist a bridge $\alpha$ and a bridge mapping $\psi_{\alpha}: A_{0} \rightarrow Y$ which has an extension $\psi_{\alpha}^{\prime}: A_{0} \cup A^{m} \rightarrow Y$.

First suppose $\operatorname{dim} X \leqq m$. Then there exists a refinement $\beta$ of $\alpha$ with $\operatorname{dim} B \leqq m$. Let $p_{\beta \alpha}: B \rightarrow A$ be a simplicial projection, then $p_{\beta \alpha}(B) \subset A_{0} \cup A^{m}$. It follows from (2.1) and its proof that $\beta$ is a bridge with $\psi_{\beta}=\psi_{\alpha} p_{\beta \alpha} \mid B_{0}$ as a bridge mapping for $f$. Let $\phi_{\beta}: X \rightarrow B$ be a canonical mapping of $\beta$, then the mappings $f$ and $\psi_{\beta} \phi_{\beta} \mid X_{0}$ are homotopic. Since $\psi_{\beta} \phi_{\beta} \mid X_{0}$ has an extension $\psi_{\alpha}^{\prime} p_{\beta \alpha} \phi_{\beta}: X \rightarrow Y$, it follows from Borsuk's theorem [13, p. 86] that $f$ has an extension $f^{\prime}: X \rightarrow Y$ and the particular case of our theorem is proved.

To the general case when $\operatorname{dim} \mathrm{Cl}\left(X-X_{0}\right) \leqq m$, we first prove an auxiliary assertion: For an arbitrary mapping $\rho: X \rightarrow A_{0} \cup A^{p+1}(p \geqq m)$ such that $\rho\left(X_{0}\right) \subset A_{0}$, there exists a mapping $\theta: X \rightarrow A_{0} \cup A^{p}$ with $\theta(x)=\rho(x)$ for each $x \in \rho^{-1}\left(A_{0} \cup A^{p}\right)$.

Let $\sigma_{1}, \sigma_{2}, \cdots, \sigma_{q}$ be the open $(p+1)$-simplexes of $A^{p+1}-A_{0}$. Let

$$
G_{i}=\rho^{-1}\left(\sigma_{i}\right) \subset X-X_{0} \quad(i=1,2, \cdots, q) ;
$$

then $G_{i}$ is an open set of $X$ with $\operatorname{dim} \bar{G}_{i} \leqq m\left[14\right.$, p. 14]. Let $F_{i}=\bar{G}_{i}-G_{i}$, then $\rho\left(F_{i}\right) \subset \partial \sigma_{i}$. Since $\partial \sigma_{i}$ is a $p$-sphere and $p \geqq m$, the conditions of our theorem are satisfied by $X=\bar{G}_{i}, X_{0}=F_{i}, Y=\partial \sigma_{i}$, and $n=0$. By the particular case proved above, the partial mapping $\rho \mid F_{i}$ has an extension $\rho_{i}^{\prime}: \bar{G}_{i} \rightarrow \partial \sigma_{i}$. The mapping $\theta: X \rightarrow A_{0} \cup A^{p}$ stated in our assertion is given by

$$
\theta(x)=\left\{\begin{array}{lr}
\rho_{i}^{\prime}(x) & \left(x \in G_{i}, i=1,2, \cdots, q\right), \\
\rho(x) & \left(x \in X-\cup G_{i}\right) .
\end{array}\right.
$$

Since $A$ is finite, we may apply the auxiliary assertion to the canonical mapping $\phi_{\alpha}: X \rightarrow A$ successively. Then follows the existence of a mapping $\phi_{\alpha}^{\prime}: X \rightarrow A_{0} \cup A^{m}$ with $\phi_{\alpha}^{\prime}(x)=\phi_{\alpha}(x)$ for each $x \in \phi_{\alpha}^{-1}\left(A_{0} \cup A^{m}\right)$. Since $f$ and $\psi_{\alpha} \phi_{\alpha} \mid X_{0}$ are homotopic and $\psi_{\alpha}^{\prime} \phi_{\alpha}^{\prime}: X \rightarrow Y$ is an extension of $\psi_{\alpha} \phi_{\alpha} \mid X_{0}$, it follows from Borsuk's theorem [13, p. 86] that $f$ has an extension $f^{\prime}: X \rightarrow Y$.

Last, if $X$ is metric separable with $\operatorname{dim}\left(X-X_{0}\right) \leqq m$, then our theorem follows from (3.3) and the $m$-extensibility of $f$. Q.E.D.

(5.2) If $Y$ is $r$-simple for each $r<m$ and

$$
H^{r}\left(X \bmod X_{0}, \pi^{r}\right)=0 \quad(0<r<m, r \neq n),
$$

then every mapping $f: X_{0} \rightarrow Y$ is n-extensible and $f$ has an extension $f^{\prime}: X \rightarrow Y$ if and only if its $(n+1)$-dimensional obstruction set $\Omega^{n+1}(f)$ contains the zero element of the group $H^{n+1}\left(X \bmod X_{0}, \pi^{n}\right)$.

Proof. The first part of the theorem is contained in (4.4), and the second part follows from (4.3) and (5.1). Q.E.D.

As a corollary of $(5.2)$ we state the following generalization of a classical theorem [13 II, p. 522].

(5.3) If $Y$ is $r$-simple and $H^{r+1}\left(X \bmod X_{0}, \pi^{r}\right)=0$ for each $0<r<m$, then every mapping $f: X_{0} \rightarrow Y$ has an extension $f^{\prime}: X \rightarrow Y$. 
Setting $Y=X_{0}$, we obtain a sufficient condition for retraction as follows. Let $X$ be a metric separable space, and $X_{0} \subset X$ be a connected compact ANR with $\operatorname{dim}\left(X-X_{0}\right) \leqq m$.

(5.4) $X_{0}$ is a retract of $X$, if $X_{0}$ is $r$-simple and $H^{r+1}\left(X \bmod X_{0}, \pi^{r}\right)=0$ for each $0<r<m$, where $\pi^{r}=\pi^{r}\left(X_{0}\right)$.

More generally, the following generalization of a theorem of Borsuk $[4, \mathrm{p}$. 166] is an immediate consequence of (3.3) and (4.4).

(5.5) If $X_{0}$ is $r$-simple and $H^{r+1}\left(X \bmod X_{0}, \pi^{r}\right)=0$ for each $0<r<n$, where $\pi^{r}=\pi^{r}\left(X_{0}\right)$, then there exist a closed set $X_{1}$ of $X$ contained in $X-X_{0}$ with $\operatorname{dim} X_{1}<m-n$ and a retraction $\theta: X-X_{1} \rightarrow X_{0}$.

6. $n$-homotopy. In the $\S \S 6,7,8$, we shall assume (1) $X$ to be a normal space, (2) $Y$ to be a connected ANR, and (3) either $X$ or $Y$ to be compact. The bridge theorems in $\$ 2$ hold with $X_{0}=X$.

Let $f_{0}, f_{1}: X \rightarrow Y$ be two given mappings. According to the bridge existence theorem (2.2), there exists a bridge $\alpha_{i}$ for $f_{i}(i=0,1)$; and by the bridge refinement theorem (2.1), every common refinement is a bridge for both $f_{0}$ and $f_{1}$. Such coverings of $X$ are called the bridges for the pair $\left(f_{0}, f_{1}\right)$. Then we have proved the $f$ sllowing bridge theorem.

(6.1) The set $\mathfrak{B}_{f_{0}, f_{1}}$ of all bridges for a given pair $\left(f_{0}, f_{1}\right)$ of mappings is residual (and hence cofinal) in the directed set $\subseteq$ of all coverings of $X$.

Two mappings $f_{0}, f_{1}: X \rightarrow Y$ are said to be $n$-homotopic, if there exists a bridge $\alpha$ for the pair $\left(f_{0}, f_{1}\right)$ with bridge mappings $\psi_{i}: A \rightarrow Y$ for $f_{i}(i=0,1)$ such that $\psi_{0}=\psi_{1}$ on the $n$-dimensional skeleton $A^{n}$ of the geometric nerve $A$ of the covering $\alpha$. Since $Y$ is arcwise connected, the following statement is trivial.

(6.2) Every pair of mappings $f_{0}, f_{1}: X \rightarrow Y$ are 0 -homotopic.

The following statement is an obvious result of the definition.

(6.3) Homotopic mappings are $n$-homotopic for every $n \geqq 0$.

The relation of being $n$-homotopic is clearly reflexive, symmetric, and transitive: hence the mappings $f: X \rightarrow Y$ are divided into classes by this equivalence relation, which are called the $n$-homotopy classes. By (6.3), each homotopy class in the sense of Brouwer is contained in one and the same $n$-homotopy class.

For a given pair of mappings $f_{0}, f_{1}: X \rightarrow Y$, the least upper bound of the set of integers $n$ such that they are $n$-homotopic is called the homotopy index of the pair $\left(f_{0}, f_{1}\right)$. From (6.3), we deduce the following statement.

(6.4) The homotopy index of a pair of mappings $f_{0}, f_{1}: X \rightarrow Y$ depends only on the homotopy classes of $f_{0}$ and $f_{1}$.

Some set-theoretic meaning of $n$-homotopy is shown by the following theorem.

(6.5) If $X$ be metric separable with $\operatorname{dim} X \leqq m$ and $f_{0}, f_{1}: X \rightarrow Y$ be $n$-homotopic mappings, then there exists a closed set $X_{1}$ of $X$ such that $\operatorname{dim} X_{1}<m-n$ and the partial mappings $f_{i} \mid X-X_{1}(i=0,1)$ are homotopic. 
Proof. By definition, there exists a bridge $\alpha$ for the pair $\left(f_{0}, f_{1}\right)$ with bridge mappings $\psi_{i}: A \rightarrow Y$ for $f_{i}(i=0,1)$ such that $\psi_{0}=\psi_{1}$ on $A^{n}$. Let $\phi_{\alpha}: X \rightarrow A$ be a canonical mapping of $\alpha$. By an auxiliary assertion given in the proof of (3.3), there exist a closed set $X_{1} \subset X$ with $\operatorname{dim} X_{1}<m-n$ and a homotopy $h_{t}: X-X_{1} \rightarrow A \quad(0 \leqq t \leqq 1)$ such that $h_{0}=\phi_{\alpha} \mid X-X_{1}, h_{1}\left(X-X_{1}\right) \subset A^{n}$, and $h_{t}(x)=\phi_{\alpha}(x)$ for each $x \in \phi_{\alpha}^{-1}\left(A^{n}\right)$ and each $0 \leqq t \leqq 1$. Our theorem follows from the three facts: (i) $\psi_{0} h_{1}=\psi_{1} h_{1}$ on $X-X_{1}$; (ii) $f_{0} \mid X-X_{1}$ and $\psi_{0} h_{1}$ are homotopic; and, (iii) $f_{1} \mid X-X_{1}$ and $\psi_{1} h_{1}$ are homotopic. Q.E.D.

7. Separation sets. In the present section, we shall assume an additional condition that $Y$ be $n$-simple. Let $\pi^{n}=\pi^{n}(Y)$.

Let $f_{0}, f_{1}: X \rightarrow Y$ be a given pair of mappings. We are going to define the $n$-dimensional separation set $\Delta^{n}\left(f_{0}, f_{1}\right)$ in $H^{n}\left(X, \pi^{n}\right)$ as follows.

If $f_{0}, f_{1}$ are not $(n-1)$-homotopic, we define $\Delta^{n}\left(f_{0}, f_{1}\right)$ to be the empty set. Now suppose $f_{0}, f_{1}$ to be $(n-1)$-homotopic. Then there exists a bridge $\alpha$ for the pair $\left(f_{0}, f_{1}\right)$ with bridge mappings $\psi_{i}: A \rightarrow Y$ for $f_{i}(i=0,1)$ such that $\psi_{0}=\psi_{1}$ on $A^{n-1}$. According to Eilenberg [9, p. 240], $\psi_{0}$ and $\psi_{1}$ determine a cocycle

$$
d^{n}\left(\psi_{0}, \psi_{1}\right)=\sum_{i}\left(\psi_{0}, \psi_{1}, \sigma_{i}^{n}\right) \sigma_{i}^{n}
$$

where $\left(\psi_{0}, \psi_{1}, \sigma_{i}^{n}\right)$ is an element of $\pi^{n} \cdot d^{n}\left(\psi_{0}, \psi_{1}\right)$ represents an element $\delta^{n}\left(\psi_{0}, \psi_{1}\right)$ of $H^{n}\left(X, \pi^{n}\right)$, called a separation element of $\left(f_{0}, f_{1}\right) . \Delta^{n}\left(f_{0}, f_{1}\right)$ is defined to be the set of all separation elements of $\left(f_{0}, f_{1}\right)$ in $H^{n}\left(X, \pi^{n}\right)$.

The following statements are trivial.

(7.1) The mappings $f_{0}, f_{1}: X \rightarrow Y$ are $(n-1)$-homotopic if and only if $\Delta^{n}\left(f_{0}, f_{1}\right)$ is nonempty.

(7.2) The $n$-dimensional separation set $\Delta^{n}\left(f_{0}, f_{1}\right)$ of the mappings $f_{0}, f_{1}: X \rightarrow Y$ depends only on the homotopy classes of $f_{0}$ and $f_{1}$.

(7.3) Fundamental homotopy Lemma. The mappings $f_{0}, f_{1}: X \rightarrow Y$ are $n$-homotopic if and only if $\Delta^{n}\left(f_{0}, f_{1}\right)$ contains the zero element of $H^{n}\left(x, \pi^{n}\right)$.

Proof. Necessity. Suppose $f_{0}, f_{1}$ to be $n$-homotopic. Then there exists a bridge $\alpha$ for $\left(f_{0}, f_{1}\right)$ with bridge mappings $\psi_{i}: A \rightarrow Y(i=0,1)$ respectively for $f_{i}$ such that $\psi_{0}=\psi_{1}$ on $A^{n}$. The cocycle $d^{n}\left(\psi_{0}, \psi_{1}\right)=0$, and hence $\Delta^{n}\left(f_{0}, f_{1}\right)$ contains the zero element.

Sufficiency. Suppose that $\Delta^{n}\left(f_{0}, f_{1}\right)$ contains the zero element of $H^{n}\left(x, \pi^{n}\right)$. Then there exist a bridge $\alpha$ for $\left(f_{0}, f_{1}\right)$ and bridge mappings $\psi_{i}: A \rightarrow Y$ for $f_{i}$ $(i=0,1)$ such that $\psi_{0}=\psi_{1}$ on $A^{n-1}$ and the cocycle $d^{n}\left(\psi_{0}, \psi_{1}\right)$ represents the zero element of $H^{n}\left(X, \pi^{n}\right)$. By the definition of Cech cohomology groups ( $\left.\$ 1\right)$, there exists a refinement $\beta$ of $\alpha$ such that the cocycle $p_{\beta \alpha}^{*}\left(d^{n}\left(\psi_{0}, \psi_{1}\right)\right)$ of $B$ induced by a simplicial projection $p_{\beta \alpha}: B \rightarrow A$ cobounds in $B$. It follows from (2.1) as well as its proof that $\beta$ is a bridge for $\left(f_{0}, f_{1}\right)$ and $\psi_{i} p_{f_{\alpha}}: B \rightarrow Y(i=0,1)$ are bridge mappings for $f_{i}$. Obviously $\psi_{0} p_{\beta \alpha}=\psi_{1} p_{\beta \alpha}$ on $B^{n-1}$ and

$$
d^{n}\left(\psi_{0} p_{\beta \alpha}, \psi_{1} p_{\beta \alpha}\right)=p_{\beta \alpha}^{*}\left(d^{n}\left(\psi_{0}, \psi_{1}\right)\right) .
$$


Since $d^{n}\left(\psi_{0} p_{\beta \alpha}, \psi_{1} p_{\beta \alpha}\right)$ cobounds, it follows from the first homotopy theorem of Eilenberg $\left[9\right.$, p. 240] that $\psi_{0} p_{\beta \alpha} \mid B^{n}$ and $\psi_{1} p_{\beta \alpha} \mid B^{n}$ are homotopic. Hence, by Borsuk's theorem [13, p. 86] there is a mapping $\psi_{1}^{\prime}: B \rightarrow Y$ such that $\psi_{0} p_{\beta \alpha}=\psi_{1}^{\prime}$ on $B^{n}$ and $\psi_{1} p_{\beta \alpha} \simeq \psi_{1}^{\prime}$. One can see clearly from the definition that $\psi_{1}^{\prime}$ is a bridge mapping for $f_{1}$; hence $f_{0}, f_{1}$ are $n$-homotopic. Q.E.D.

The following theorem is an immediate consequence of (7.1) and (7.3).

(7.4) If $Y$ is $r$-simple and $H^{r}\left(X, \pi^{r}\right)=0$ for each $0<r \leqq n$, then every pair of mappings $f_{0}, f_{1}: X \rightarrow Y$ are $n$-homotopic.

Now let $X^{\prime}$ be another normal space and $g: X^{\prime} \rightarrow X$ be a given mapping. Denote by $g^{*}: H^{n}\left(X, \pi^{n}\right) \rightarrow H^{n}\left(X^{\prime}, \pi^{n}\right)$ the homomorphism induced by $g(\$ 1)$.

(7.5) If $f_{0}, f_{1}: X \rightarrow Y$ be $(n-1)$-homotopic, then so are $f_{0} g, f_{1} g: X^{\prime} \rightarrow Y$ and $\Delta^{n}\left(f_{0} g, f_{1} g\right)$ contains the image $g^{*}\left(\Delta^{n}\left(f_{0}, f_{1}\right)\right)$ under $g^{*}$.

Proof. Let $\xi \in \Delta^{n}\left(f_{0}, f_{1}\right)$ be an arbitrary element. By definition, there exist a bridge $\alpha$ for $\left(f_{0}, f_{1}\right)$ and bridge mappings $\psi_{i}: A \rightarrow Y$ for $f_{i}(i=0,1)$ such that $\psi_{0}=\psi_{1}$ on $A^{n-1}$ and the cocycle $d^{n}\left(\psi_{0}, \psi_{1}\right)$ represents $\xi$. Let the members of $\alpha$ be the open sets $a_{1}, a_{2}, \cdots, a_{r}$; and denote by $\alpha^{\prime}=\left\{a_{1}^{\prime}, a_{2}^{\prime}, \cdots, a_{r}^{\prime}\right\}$ the covering of $X^{\prime}$ which consists of the open sets $a_{i}^{\prime}=g^{-1}\left(a_{i}\right)$. Let $\phi: X \rightarrow A$ and $\phi^{\prime}: X^{\prime} \rightarrow A^{\prime}$ be canonical mappings, and $t: A^{\prime} \rightarrow A$ denote the simplicial mapping determined by the correspondence $a_{i}^{\prime} \rightarrow a_{i}$. Since $\psi_{i}$ is a bridge mapping for $f_{i}$, we have $\psi_{i} \phi \simeq f_{i}(i=0,1)$. Hence, by $(4.5)$,

$$
\psi_{i} t \phi^{\prime} \simeq \psi_{i} \phi g \simeq f_{i} g \quad(i=0,1) .
$$

Therefore, $\alpha^{\prime}$ is a bridge for the pair $\left(f_{0} g, f_{1} g\right)$ with $\psi_{i} t: A^{\prime} \rightarrow Y$ as bridge mappings for $f_{i} g(i=0,1)$. Since $\psi_{0} t=\psi_{1} t$ on $A^{\prime n-1}, f_{0} g$ and $f_{1} g$ are $(n-1)$-homotopic. Since clearly

$$
\delta^{n}\left(\psi_{0} t, \psi_{1} t\right)=g^{*}\left(\delta^{n}\left(\psi_{0}, \psi_{1}\right)\right),
$$

we deduce the inclusion $\Delta^{n}\left(f_{0} g, f_{1} g\right) \supset g^{*}\left(\Delta^{n}\left(f_{0}, f_{1}\right)\right)$. Q.E.D.

8. General homotopy theorems. In this section, we assume $\operatorname{dim} X \leqq m$, where $m$ is a finite integer. Let $\pi^{r}=\pi^{r}(Y)$.

(8.1) If $Y$ is $r$-simple and $H^{r}\left(X, \pi^{r}\right)=0$ for each $n<r \leqq m$, then any two n-homotopic mappings $f_{0}, f_{1}: X \rightarrow Y$ are homotopic.

Proof. By the recurrent applications of (7.1) and (7.3) one can see that $f_{0}, f_{1}$ are $m$-homotopic. Then there exist a bridge $\alpha$ for $\left(f_{0}, f_{1}\right)$ and bridge mappings $\psi_{i}: A \rightarrow Y$ for $f_{i}(i=0,1)$ such that $\psi_{0}=\psi_{1}$ on $A^{m}$. Since $\operatorname{dim} X \leqq m$, there exists a refinement $\beta$ of $\alpha$ with $\operatorname{dim} B \leqq m$. Let $p_{\beta \alpha}: B \rightarrow A$ be a simplicial projection, then $p_{\beta \alpha}(B) \subset A^{m}$. Since, according to (2.1) and its proof, $\beta$ is a bridge for $\left(f_{0}, f_{1}\right)$ with $\psi_{i} p_{\beta \alpha}: B \rightarrow Y$ as bridge mappings for $f_{i}(i=0,1)$, we have

$$
\psi_{i} p_{\beta \alpha} \phi_{\beta} \simeq f_{i} \quad(i=0,1)
$$

for any canonical mapping $\phi_{B}: X \rightarrow B$ of $\beta$. Since $\psi_{n}=\psi_{1}$ on $A^{m}$ and $p_{\beta \alpha}(B) \subset A^{m}$, 
we have $\psi_{0} p_{\beta \alpha} \phi_{\beta}=\psi_{1} p_{\beta \alpha} \phi_{\beta}$. This proves $f_{0} \simeq f_{1}$. Q.E.D.

(8.2) If $Y$ is $r$-simple for each $r \leqq m$ and if $H^{r}\left(X, \pi^{r}\right)=0$ for each $0<r \leqq m$ except $r=n$, then any two mappings $f_{0}, f_{1}: X \rightarrow Y$ are $(n-1)$-homotopic and they are homotopic if and only if their $n$-dimensional separation set $\Delta^{n}\left(f_{0}, f_{1}\right)$ contains the zero element of $H^{n}\left(X, \pi^{n}\right)$.

Proof. The first part of the theorem is contained in (7.4), and the second part follows from (7.3) and (8.1).

As corollaries of (8.2), we state the following generalizations of two theorems of Hurewicz [12 II].

(8.3) If $Y$ is $r$-simple and $H^{r}\left(X, \pi^{r}\right)=0$ for each $0<r \leqq m$, then every mapping $f: X \rightarrow Y$ is null-homotopic.

If we set $X=Y$, then $X$ becomes a connected compact ANR with $\operatorname{dim} X \leqq m$.

(8.4) $X$ is contractible to a point, if and only if $X$ be $r$-simple and $H^{r}\left(X, \pi^{r}\right)$ $=0$ for each $0<r \leqq m$, where $\pi^{r}=\pi^{r}(X)$.

The following statement is an interesting result of (8.4).

(8.5) A necessary and sufficient condition that $\pi^{r}(X)=0$ for each $0<r \leqq m$ is that $X$ be $r$-simple and $H^{r}\left(X, \pi^{r}\right)=0$ for each $0<r \leqq m$, where $\pi^{r}=\pi^{r}(X)$.

Let $X$ and $X_{0} \subset X$ be connected compact ANR, then the following sufficient condition for deformation retracts [3] follows easily from (5.4) and (8.2).

(8.6) $X_{0}$ is a deformation retract of $X$, if the following conditions are satisfied:

(i) $X_{0}$ is $r$-simple and $H^{r+1}\left(X \bmod X_{0}, \pi^{r}\left(X_{0}\right)\right)=0$ for each $0<r<m$.

(ii) $X$ is $r$-simple and $H^{r}\left(X, \pi^{r}(X)\right)=0$ for each $0<r \leqq m$.

\section{The CASE $X$ COMpact AND $\pi^{r}(Y)=0(r<n)$}

9. Characteristic element. Throughout the remainder of this work, we assume that $X$ be a compact Hausdorff (and hence normal) space and $Y$ be a connected ANR with $\pi^{r}(Y)=0$ for each $r<n$. If $n>1$ the latter condition implies $\pi^{1}(Y)=0$ and hence the $i$-simplicity of $Y$ for all $i$. If $n=1$ we assume the $i$-simplicity of $Y$ for each $i \leqq m$, where $m$ is an integer to be specified in the sequel. Choose a fixed point $y_{0} \in Y$ as the base point for the homotopy groups $\pi^{r}=\pi^{r}(Y)$.

Let $X_{0}$ denote a closed (and hence compact) subset of $X$, and let $f: X_{0} \rightarrow Y$ denote a given mapping.

Let $\alpha$ be an arbitrary bridge for $f$ with $\psi_{\alpha}: A_{0} \rightarrow Y$ as a bridge mapping. Since $\pi^{r}(Y)=0$ for each $r<n$, we may assume that $\psi_{\alpha}\left(A_{0}^{n-1}\right)=y_{0}$. For each oriented $n$-simplex $\sigma_{i}^{n} \in A_{0}$, the partial mapping $\psi_{\alpha} \mid \bar{\sigma}_{i}^{n}$ determines an element $\left(\psi_{\alpha}, \sigma_{i}^{n}\right)$ of the homotopy group $\pi^{n}=\pi^{n}(Y)$. Since $\psi_{\alpha}$ is defined throughout $A_{0}$, the $n$-chain

$$
k^{n}\left(\psi_{\alpha}\right)=\sum_{i}\left(\psi_{\alpha}, \sigma_{i}^{n}\right) \sigma_{i}^{n}
$$

is clearly a cocycle of $A_{0}$. 
(9.1) All the possible cocycles $k^{n}\left(\psi_{\alpha}\right)$ represent a unique element $\kappa^{n}(f)$ of $H^{n}\left(X_{0}, \pi^{n}\right)$, called the characteristic element of the mapping $f: X_{0} \rightarrow Y$.

Proof. Let $k^{n}\left(\psi_{\beta}\right)$ be another such cocycle defined by the bridge $\beta$ and the bridge mapping $\psi_{\beta}: B_{0} \rightarrow Y$ with $\psi_{\beta}\left(B_{0}^{n-1}\right)=y_{0}$.

According to the bridge homotopy theorem (2.4), there exists a common refinement $\gamma$ of $\alpha$ and $\beta$ such that $\psi_{\alpha} p_{\gamma \alpha} \mid C_{0}$ and $\psi_{\beta} p_{\gamma \beta} \mid C_{0}$ are homotopic, where $p_{\gamma \alpha}: C \rightarrow A$ and $p_{\gamma \beta}: C \rightarrow B$ are arbitrary simplicial projections.

Since $\psi_{\alpha}\left(A_{0}^{n-1}\right)=y_{0}=\psi_{\beta}\left(B_{0}^{n-1}\right)$ and $p_{\gamma \alpha}, p_{\gamma \beta}$ are simplicial, we have

$$
\psi_{\alpha} p_{\gamma \alpha}\left(C_{0}^{n-1}\right)=y_{0}=\psi_{\beta} p_{\gamma \phi}\left(C_{0}^{n-1}\right) \text {. }
$$

Hence, the cocycles $k^{n}\left(\psi_{\alpha} p_{\gamma \alpha}\right)$ and $k^{n}\left(\psi_{\beta} p_{\gamma \beta}\right)$ are defined.

Since $\psi_{\alpha} p_{\gamma \alpha} \mid C_{0}$ and $\psi_{\beta} p_{\gamma \beta} \mid C_{0}$ are homotopic, and since $\pi^{r}(Y)=0$ for each $r<n$, we have $\psi_{\alpha} p_{\gamma \alpha}\left|C_{0} \simeq \psi_{\beta} p_{\gamma \beta}\right| C_{0}$ rel. $C_{0}^{n-2}$. Hence it follows from the first homotopy theorem of Eilenberg [9, p. 240] that $k^{n}\left(\psi_{\alpha} p_{\gamma \alpha}\right)$ and $k^{n}\left(\psi_{\beta} p_{\gamma \beta}\right)$ are cohomologous in $C_{0}$. It is obvious that

$$
k^{n}\left(\psi_{\alpha} p_{\gamma \alpha}\right)=p_{\gamma \alpha}^{*}\left(k^{n}\left(\psi_{\alpha}\right)\right), \quad k^{n}\left(\psi_{\beta} p_{\gamma \beta}\right)=p_{\gamma \beta}^{*}\left(k^{n}\left(\psi_{\beta}\right)\right),
$$

where $p_{\gamma \alpha}^{*}, p_{\gamma \beta}^{*}$ denote the homomorphisms of cocycles induced by the simplicial projections $p_{\gamma \alpha}: C \rightarrow A$ and $p_{\gamma \beta}: C \rightarrow B$. Hence, it results from the definition of Čech cohomology groups $(\$ 1)$ that $k^{n}\left(\psi_{\alpha}\right)$ and $k^{n}\left(\psi_{\beta}\right)$ represent the same element of $H^{n}\left(X_{0}, \pi^{n}\right)$. This completes the proof. Q.E.D.

The following statement is obvious from the definition.

(9.2) Homotopic mappings have the same characteristic element.

The following statement can be easily proved for an arbitrary compact Hausdorff space $X_{0}^{\prime}$.

(9.3) If $f: X_{0} \rightarrow Y$ and $g: X_{0}^{\prime} \rightarrow X_{0}$ be mappings, then $\kappa^{n}(f g)=g^{*}\left(\kappa^{n}(f)\right)$, where $g^{*}: H^{n}\left(X_{0}, \pi^{n}\right) \rightarrow H^{n}\left(X_{0}^{\prime}, \pi^{n}\right)$ denotes the homomorphism induced by $g$.

(9.4) The obstruction set $\Omega^{n+1}(f)$ consists of a single element $\omega^{n+1}(f)=\delta \kappa^{n}(f)$, called the obstruction element of the mapping $f: X_{0} \rightarrow Y$.

Proof. First, let us prove that $\delta \kappa^{n}(f) \in \Omega^{n+1}(f)$. By definition, there exist a bridge $\alpha$ for $f$ and a bridge mapping $\psi_{\alpha}: A_{0} \rightarrow Y$ such that $\psi_{\alpha}\left(A_{0}^{n-1}\right)=y_{0}$ and the cocycle $k^{n}\left(\psi_{\alpha}\right)$ of $A_{0}$ represents the element $\kappa^{n}(f) . \psi_{\alpha}$ has a trivial extension $\psi_{\alpha}^{\prime}: A_{0} \cup A^{n} \rightarrow Y$ defined by taking

$$
\psi_{\alpha}^{\prime}(\xi)=\left\{\begin{array}{lr}
\psi_{\alpha}(\xi) & \left(\xi \in A_{0}\right), \\
y_{0} & \left(\xi \in A^{n}-A_{0}\right) .
\end{array}\right.
$$

It is not difficult to verify the fact that $w^{n+1}\left(\psi_{\alpha}^{\prime}\right)=\delta k^{n}\left(\psi_{\alpha}\right)$; hence, it follows that $\delta \kappa^{n}(f)$ is an obstruction element of $f$.

Second, suppose $\omega^{n+1}$ be an arbitrary element of $\Omega^{n+1}(f)$. Then there exist a bridge $\alpha$ and a bridge mapping $\psi_{\alpha}: A_{0} \rightarrow Y$ for $f$ such that $\psi_{\alpha}$ has an extension $\psi_{\alpha}^{\prime}: A_{0} \cup A^{n} \rightarrow Y$ and the cocycle $w^{n+1}\left(\psi_{\alpha}^{\prime}\right)$ of $A-A_{0}$ is a representative of the element $\omega^{n+1}$. Since $\pi^{r}(Y)=0$ for each $r<n$, there exists a bridge mapping 
$\theta_{\alpha}: A_{0} \rightarrow Y$ for $f$ such that $\theta_{\alpha} \simeq \psi_{\alpha}$ and $\theta_{\alpha}\left(A_{0}^{n-1}\right)=y_{0} . \theta_{\alpha}$ has a trivial extension $\theta_{\alpha}^{\prime}: A_{0} \cup A^{n} \rightarrow Y$ defined by

$$
\theta_{\alpha}^{\prime}(\xi)=\left\{\begin{array}{lr}
\theta_{\alpha}(\xi) & \left(\xi \in A_{0}\right), \\
y_{0} & \left(\xi \in A^{n}-A_{0}\right) .
\end{array}\right.
$$

According to a theorem of Eilenberg [9, p. 241], the cocycles $w^{n+1}\left(\psi_{\alpha}^{\prime}\right)$ and $w^{n+1}\left(\theta_{\alpha}^{\prime}\right)$ are cohomologous in $A-A_{0}$. On the other hand, we have $w^{n+1}\left(\theta_{\alpha}^{\prime}\right)$ $=\delta k^{n}\left(\theta_{\alpha}\right)$; hence $\omega^{n+1}=\delta \kappa^{n}(f)$. This completes the proof. Q.E.D.

Now, let us consider the case $X_{0}=X$. For a given pair of mappings $f_{0}, f_{1}$ : $X \rightarrow Y$, the following theorem can be easily proved.

(9.5) The separation set $\Delta^{n}\left(f_{0}, f_{1}\right)$ consists of a single element $\delta^{n}\left(f_{0}, f_{1}\right)$ $=\kappa^{n}\left(f_{0}\right)-\kappa^{n}\left(f_{1}\right)$, called the separation element of the mappings $f_{0}, f_{1}: X \rightarrow Y$.

10. Extension theorems. An element of $H^{n}\left(X_{0}, G\right)$ is said to be extendible, if it is contained in the image $\eta\left(H^{n}(X, G)\right)$ under the homomorphism $\eta$ of the cohomology sequence. lent:

(10.1) For a given mapping $f: X_{0} \rightarrow Y$ the following statements are equiva-

(i) $f$ is $(n+1)$-extensible.

(ii) $\omega^{n+1}(f)=0$.

(iii) $\kappa^{n}(f)$ is extendible.

Proof. The equivalence of (i) and (ii) follows from (4.3) and (9.4); and that of (ii) and (iii) follows from the exactness of the cohomology sequence. Q.E.D.

The following statement, which is an immediate result of (10.1), gives us a weaker sufficient condition than the condition $H^{n+1}\left(X \bmod X_{0}, \pi^{n}\right)=0$ for every mapping $f: X_{0} \rightarrow Y$ to be $(n+1)$-extensible.

(10.2) If all elements of $H^{n}\left(X_{0}, \pi^{n}\right)$ are extendible, then every mapping $f: X_{0} \rightarrow Y$ is $(n+1)$-extensible.

In particular, we state the important special case as a corollary of (10.2).

(10.3) If $H^{n}\left(X_{0}, \pi^{n}\right)=0$, then each mapping $f: X_{0} \rightarrow Y$ is $(n+1)$-extensible.

In the remainder of this section, let $m$ be a finite positive integer. If $X$ is metric separable, we assume $\operatorname{dim}\left(X-X_{0}\right) \leqq m$; otherwise, we shall assume $\left(^{3}\right)$ the stronger condition $\operatorname{dim} \mathrm{Cl}\left(X-X_{0}\right) \leqq m$. (10.1).

The following extension theorem is an obvious consequence of (5.1) and

(10.4) If $H^{r+1}\left(X \bmod X_{0}, \pi^{r}\right)=0$ for each $n<r<m$, then, for a given mapping $f: X_{0} \rightarrow Y$, the following statements are equivalent:

(i) $f$ has an extension $f^{\prime}: X \rightarrow Y$.

(ii) $\omega^{n+1}(f)=0$.

(iii) $\kappa^{n}(f)$ is extendible.

As a corollary of (10.4) we state the following generalization of a classical theorem [12 II, p. 522]. 
(10.5) If $H^{n}\left(X_{0}, \pi^{n}\right)=0$ and $H^{r+1}\left(X \bmod X_{0}, \pi^{r}\right)=0$ for each $n<r<m$, then every mapping $f: X_{0} \rightarrow Y$ has an extension $f^{\prime}: X \rightarrow Y$.

11. Homotopy theorems. In the present section, we take $X_{0}=X$. The following theorem results from (7.3) and (9.5).

(11.1) For given mappings $f_{0}, f_{1}: X \rightarrow Y$ the following statements are equivalent:

(i) $f_{0}, f_{1}$ are $n$-homotopic.

(ii) $\delta^{n}\left(f_{0}, f_{1}\right)=0$.

(iii) $\kappa^{n}\left(f_{0}\right)=\kappa^{n}\left(f_{1}\right)$.

Then (8.2) becomes the following theorem.

(11.2) If $\operatorname{dim} X \leqq m$ and $H^{r}\left(X, \pi^{r}\right)=0$ for each $n<r \leqq m$, then the following statements are equivalent:

(i) $f_{0}, f_{1}$, are homotopic.

(ii) $\delta^{n}\left(f_{0}, f_{1}\right)=0$.

(iii) $\kappa^{n}\left(f_{0}\right)=\kappa^{n}\left(f_{1}\right)$.

(11.3) Existence THEOREM. If $\operatorname{dim} X \leqq m$ and $H^{r+1}\left(X, \pi^{r}\right)=0$ for each $n<r<m$, then for each element $\xi \in H^{n}\left(X, \pi^{n}\right)$ there exists a mapping $f: X \rightarrow Y$ with its characteristic element $\kappa^{n}(f)=\xi$.

Proof There exist a covering $\alpha_{1}$ of $X$ and a cocycle $c^{n}=\sum_{i} c_{i} \sigma_{i}^{n}$ on the nerve $A_{1}$ of $\alpha_{1}$ which represents the element $\xi$, where $c_{i} \in \pi^{n}$.

Define a mapping $\theta_{1}: A_{1}^{n+1} \rightarrow Y$ described as follows. First, let $\theta_{1}\left(A_{1}^{n-1}\right)=y_{0}$. Since $c_{i} \in \pi^{n}$, one can define $\theta_{1}$ over $A_{1}^{n}$ so that $\theta_{1} \mid \bar{\sigma}_{i}^{n}$ defines $c_{i}$ for each $\sigma_{i}^{n} \in A_{i}^{n}$. Since $c^{n}$ is a cocycle, for each $\sigma^{n+1} \in A_{1}$, we have

$$
\sum_{\sigma_{i}^{n} \in \partial \sigma^{n+1}} c_{i}=0 \text {. }
$$

Hence, $\theta_{1} \mid \partial \sigma^{n+1}$ is nullhomotopic and $\theta_{1}$ can be extended throughout $A_{1}^{n+1}$.

For each $(n+2)$-simplex $\sigma_{i}^{n+2}$ of $A_{1}, \theta_{1} \mid \partial \sigma_{i}^{n+2}$ determines an element $\left(\theta_{1}, \sigma_{i}^{n+2}\right)$ of $\pi^{n+1}(Y)$. The chain $c_{1}^{n+2}=\sum_{i}\left(\theta_{1}, \sigma_{i}^{n+2}\right) \sigma_{i}^{n+2}$ is a cocycle of $A_{1}$ [9, p. 237], and hence it represents an element of $H^{n+2}\left(X, \pi^{n+1}\right)$. Since $H^{n+2}\left(X, \pi^{n+1}\right)=0$, there is a refinement $\alpha_{2}$ of $\alpha_{1}$ and a simplicial projection $p: A_{2} \rightarrow A_{1}$ such that the cocycle

$$
c_{2}^{n+2}=\sum_{i}\left(\theta_{1} p, \sigma_{i}^{n+2}\right) \sigma_{i}^{n+2}, \quad \sigma_{i}^{n+2} \in A_{2},
$$

cobounds in $A_{2}$. According to the first extension theorem of Eilenberg [9, p. 239], there exists a mapping $\theta_{2}: A_{2}^{n+2} \rightarrow Y$ with $\theta_{2}=\theta_{1} p$ on $A_{2}^{n} . \theta_{2} \mid \bar{\sigma}_{i}^{n}$ determines an element $\left(\theta_{2}, \sigma_{i}^{n}\right)$ of $\pi^{n}$ for each $\sigma_{i}^{n} \in A_{2}$. The cocycle $\sum_{i}\left(\theta_{2}, \sigma_{i}^{n}\right) \sigma_{i}^{n}$ clearly represents the given element $\xi$.

By the successive use of the above argument, one can prove the existence of a covering $\alpha_{m-n}$ of $X$ and a mapping $\theta_{m-n}: A_{m-n}^{m} \rightarrow Y$ such that $\theta_{m-n}\left(A_{m-n}^{n-1}\right)$ $=y_{0}$ and the cocycle $\sum_{i}\left(\theta_{m-n}, \sigma_{i}^{n}\right) \sigma_{i}^{n}$ of $A_{m-n}$ represents the given element $\xi$. 
Since $\operatorname{dim} X \leqq m$, there is a refinement $\alpha$ of $\alpha_{m-n}$ with $\operatorname{dim} A \leqq m$. Let $p: A \rightarrow A_{m-n}$ be a simplicial projection; then $\psi_{\alpha}=\theta_{m-n} p$ is a mapping $A \rightarrow Y$ such that $\psi_{\alpha}\left(A^{n-1}\right)=y_{0}$ and the cocycle $k^{n}\left(\psi_{\alpha}\right)$ represents $\xi$. Let $\phi_{\alpha}: X \rightarrow A$ be a canonical mapping of $\alpha$, and define a mapping $f: X \rightarrow Y$ by taking $f=\psi_{\alpha} \phi_{\alpha}$; then $\alpha$ is a bridge and $\psi_{\alpha}$ a bridge mapping for $f$. Hence $\kappa^{n}(f)=\xi$. Q.E.D.

From (11.2) and (11.3) follows our generalized Hopf classification theorem stated below.

(11.4) Classification theorem. If $\operatorname{dim} X \leqq m$ and $H^{r}\left(X, \pi^{r}\right)=0$ $=H^{r+1}\left(X, \pi^{r}\right)$ for each $n<r \leqq m$, then the elements of $H^{n}\left(X, \pi^{n}\right)$ are in a (1-1)correspondence with the homotopy classes of the mappings $f: X \rightarrow Y$. The correspondence is determined by the operation $\kappa^{n}(f)$.

12. The basic element $\iota^{n}(Y)$. In addition to the conditions given at the beginning of $\S 9$, we shall assume $Y$ to be compact for the remaining sections.

The characteristic element $\kappa^{n}(\iota) \in H^{n}\left(Y, \pi^{n}\right)$ of the identity mapping $\iota: Y \rightarrow Y$ is defined to be the basic element of $Y$, denoted by $\iota^{n}(Y)$.

(12.1) The basic element $\iota^{n}(Y)=0$ if and only if $\pi^{n}(Y)=0$.

Proof. Sufficiency is trivial. To prove the necessity, let us assume $\iota^{n}(Y)$ $=0$. There exist a bridge $\alpha$ for $\iota$ which is a covering of $Y$ and a bridge $\psi_{\alpha}: A \rightarrow Y$ such that $\psi_{\alpha}\left(A^{n-1}\right)=y_{0}$ and the cocycle $c^{n}=\sum:\left(\psi_{\alpha}, \sigma_{i}^{n}\right) \sigma_{i}^{n}$ represents the zero element of $H^{n}\left(X, \pi^{n}\right)$. By passing to a refinement of $\alpha$ if necessary, we may assume that $c^{n}$ cobounds in $A$. Hence, according to the first homotopy theorem of Eilenberg [9, p. 240], $\psi_{\alpha} \mid A^{n}$ is nullhomotopic relative to $A^{n-2}$; and, therefore, there exists a mapping $\theta_{\alpha}: A \rightarrow Y$ such that $\theta_{\alpha} \simeq \psi_{\alpha}$ rel. $A^{n-2}, \theta_{\alpha}\left(A^{n}\right)$ $=y_{0} . \theta_{\alpha}$ is clearly a bridge mapping for $\iota$, that is, $\theta_{\alpha} \phi_{\alpha} \sim_{\iota}$ for each canonical mapping $\phi_{\alpha}: Y \rightarrow A$ of $\alpha$.

Now let $g: S^{n} \rightarrow Y$ be an arbitrary mapping; then we have $g \simeq \theta_{\alpha} \phi_{\alpha} g$. By the classical process of simplicial approximation, there is a sufficiently fine triangulation of $S^{n}$ (still denoted by $S^{n}$ ) such that the mapping $\phi_{\alpha} g: S^{n} \rightarrow A$ is homotopic with a simplicial mapping $\tau: S^{n} \rightarrow A$. Hence $g$ is homotopic with the constant mapping $\theta_{\alpha} \tau\left(S^{n}\right)=y_{0}$. This proves $\pi^{n}(Y)=0$. Q.E.D.

(12.2) For an arbitrary mapping $f: X_{0} \rightarrow Y$, the characteristic element $\kappa^{n}(f)$ $=f^{*}\left(\iota^{n}(Y)\right)$, where $f^{*}$ denotes the homomorphism induced by the mapping $f$.

Proof. Since $f=\imath f$, where $\iota: Y \rightarrow Y$ denotes the identity mapping, our theorem follows directly from (9.3).

By the aid of (12.2) and in the terms of the basic element $\iota^{n}(Y)$ and the induced homomorphism $f^{*}$, our generalized Hopf classification theorem (11.4) can be stated in a form which is completely analogous to the Hopf classification theorem given in the introduction.

If $Y=S^{1}$, then $\pi^{r}\left(S^{1}\right)=0$ for each $r>1$. The conditions in (11.4) are all satisfied for any compact Hausdorff space $X$ with finite dimension. Hence (11.4) includes also the following theorem of Bruschlinsky [5].

(12.3) For an arbitrary compact Hausdorff space $X$ of finite dimension, the 
elements of the 1-dimensional Cech cohomology group $H^{1}(X)$ of $X$ with integer coefficients are in a (1-1)-correspondence with the homology classes of the mappings $f: X \rightarrow S^{1}$. The correspondence is determined by the operation $f^{*}(\iota)$, where $\iota$ denotes a generator of the free cyclic group $H^{1}\left(S^{1}\right)$.

13. Homotopy type. In this section, we shall establish for connected compact ANR the theorems concerning the homotopy types [12 III, p. 124], which were proved by Eilenberg [10] only for geometric complexes. His original proof is complicated by the use of homology.

(13.1) Two connected compact absolute neighborhood retracts $X$ and $Y$ of finite dimensions are of the same homotopy type, if the following conditions are satisfied:

(i) $\pi^{r}(X)=0=\pi^{r}(Y)$ for each $r<n$.

(ii) $\pi^{n}(X)$ and $\pi^{n}(Y)$ are isomorphic.

(iii) $H^{r+1}\left(X, \pi^{r}(Y)\right)=0=H^{r+1}\left(Y, \pi^{r}(X)\right)$ for each $r>n$.

(iv) $H^{r}\left(X, \pi^{r}(X)\right)=0=H^{r}\left(Y, \pi^{r}(Y)\right)$ for each $r>n$.

If $n=1$ we replace (i) by the following:

(i) ${ }^{\prime} X$ and $Y$ are $i$-simple for each $i=1,2, \cdots, k$, where $k=\max (\operatorname{dim} X$, $\operatorname{dim} Y)$.

Proof. Let $h: \pi^{n}(X) \rightarrow \pi^{n}(Y)$ be an isomorphism onto. $h$ induces two isomorphisms onto:

$$
\begin{aligned}
& h_{x}: H^{n}\left(X, \pi^{n}(X)\right) \rightarrow H^{n}\left(X, \pi^{n}(Y)\right), \\
& h_{y}: H^{n}\left(Y, \pi^{n}(X)\right) \rightarrow H^{n}\left(Y, \pi^{n}(Y)\right) .
\end{aligned}
$$

Let $\iota^{n}(X) \in H^{n}\left(X, \pi^{n}(X)\right)$ and $\iota^{n}(Y) \in H^{n}\left(Y, \pi^{n}(Y)\right)$ denote the basic elements of $X$ and $Y$ respectively. By the existence theorem (11.3), there exist mappings $f: X \rightarrow Y$ and $g: Y \rightarrow X$ such that

$$
\begin{aligned}
& \kappa^{n}(f)=f^{*}\left(\iota^{n}(Y)\right)=h_{x}\left(\iota^{n}(X)\right), \\
& \kappa^{n}(g)=g^{*}\left(\iota^{n}(X)\right)=h_{y}^{-1}\left(\iota^{n}(Y)\right) .
\end{aligned}
$$

Since it is quite obvious that $f^{*} h_{\nu}^{-1}=h_{x}^{-1} f^{*}$, the characteristic element $\kappa^{n}(g f)$ of the mapping $g f: X \rightarrow X$ is given by

$$
\begin{aligned}
\kappa^{n}(g f) & =f^{*} g^{*}\left(\iota^{n}(X)\right)=f^{*} h_{y}^{-1}\left(\iota^{n}(Y)\right) \\
& =h_{x}^{-1} f^{*}\left(\iota^{n}(Y)\right)=h_{x}^{-1} h_{x}\left(\iota^{n}(X)\right)=\iota^{n}(X) .
\end{aligned}
$$

Hence it follows from the homotopy theorem (11.2) that $g f$ is homotopic with the identity mapping on $X$. Similarly, we prove that $f g$ is homotopic with the identity mapping on $Y$. Q.E.D.

The following statements are easy consequences of (13.1).

(13.2) A connected compact absolute neighborhood retract $Y$ of finite dimension is of the same homotopy type as $S^{n}(n>1)$ if and only if the following conditions are satisfied: 
(i) $\pi^{r}(Y)=0$ for each $r<n$.

(ii) $\pi^{n}(Y)$ is infinite cyclic.

(iii) $H^{r+1}\left(Y, \pi^{r}\left(S^{n}\right)\right)=0$ for each $r>n$.

(iv) $H^{r}\left(Y, \pi^{r}(Y)\right)=0$ for each $r>n$.

(13.3) A connected compact absolute neighborhood retract $Y$ of finite dimension is of the same homotopy type as the circle $S^{1}$ if and only if the following conditions are satisfied:

(i) $\pi^{1}(Y)$ is infinite cyclic.

(ii) $Y$ is $i$-simple for each $i=2,3, \cdots, \operatorname{dim} Y$.

(iii) $H^{r}\left(Y, \pi^{r}(Y)\right)=0$ for each $r>1$.

\section{BIBLIOGRAPHY}

1. P. Alexandroff, On the dimension of normal spaces, Proc. Royal Soc. London Ser. A vol. 189 (1947) pp. 11-39.

2. S. Banach, Théorie des operations linéaires, Warsaw, 1932.

3. K. Borsuk, Zur kombinatorischen Eigenschaften der Retrakte, Fund. Math. vol. 21 (1933) pp. $90-98$.

4. - Un théorème sur les prolongements des transformations, Fund. Math. vol. 29 (1937) pp. 161-166.

5. N. Bruschlinsky, Stetige Abbildungen und Bettische Gruppen der Dimensionszahlen 1 und 3, Math. Ann. vol. 109 (1934) pp. 525-537.

6. C. H. Dowker, Hopf's theorems for non-compact spaces, Proc. Nat. Acad. Sci. U.S.A. vol. 23 (1937) pp. 293-294.

7. S. Eilenberg, Un théorème de dualité, Fund. Math. vol. 26 (1936) pp. 280-282.

8. - On the relation between the fundamental group of a space and higher homotopy groups, Fund. Math. vol. 32 (1939) pp. 167-175.

9. - Cohomology and continuous mappings, Ann. of Math. vol. 41 (1940) pp. 231-251.

10. - Continuous mappings of infinite polyhedra, Ann. of Math. vol. 42 (1941) pp. 459-468.

11. S. Eilenberg and N. E. Steenrod, Axiomatic approach to homology theory, Proc. Nat. Acad. Sci. U.S.A. vol. 31 (1945) pp. 117-120.

12. W. Hurewicz, Beiträge zur topologie der Deformationen, I-IV, Proceedings, K. Akadamie van Wetenschappen, Amsterdam, vol. 38 (1935) pp. 112-119, 521-528; vol. 39 (1936) pp. 117$125,215-223$.

13. W. Hurewicz and H. Wallman, Dimension theory, Princeton, 1941.

14. S. Lefschetz, Algebraic topology, Amer. Math. Soc. Colloquium Publications, vol. 27, New York, 1942.

15. - Topics in topology, Princeton, 1942.

16. J. W. Tukey, Convergence and uniformity in topology, Princeton, 1940.

17. H. Whitney, On matrices of integers and combinatorial topology, Duke Math. J. vol. 3 (1937) pp. 35-45.

18. M. Wojdyslawski, Rétractes absolus et hyperespaces des continus, Fund. Math. vol. 32 (1939) pp. 184-192.

Manchester University, Manchester, ENGLaNd. 